\title{
Director Connectedness: Monitoring Efficacy and Career Prospects
}

\author{
Vincent J. Intintoli, Kathleen M. Kahle, and Wanli Zhao*
}

\begin{abstract}
We examine a specific channel through which director connectedness may improve monitoring: financial reporting quality. We find that the connectedness of independent, nonco-opted audit committee members has a positive effect on financial reporting quality and accounting conservatism. The effect is not significant for non-audit committee or co-opted audit committee members. Our results are robust to tests designed to mitigate self-selection. Consistent with connected directors being valuable, the market reacts more negatively to the deaths of highly connected directors than to the deaths of less connected directors. Better connected directors also have better career prospects, suggesting they have greater incentives to monitor.
\end{abstract}

\section{Introduction}

Conventional wisdom suggests that outside directors increase firm value, yet empirical studies provide mixed evidence regarding the effect of board independence on firm performance (Hermalin and Weisbach (1998), Bhagat and Black (1999)). One possible explanation is that directors who are conventionally independent may not be truly independent from the chief executive officer's (CEO) influence (Hwang and Kim (2009), Fracassi and Tate (2012), and Coles, Daniel, and Naveen (2014)). Another possibility is that other director characteristics affect

\footnotetext{
*Intintoli, vintint@ clemson.edu, Clemson University College of Business; Kahle (corresponding author), kkahle@eller.arizona.edu, University of Arizona Eller College of Management; and Zhao, zhaowanli@ruc.edu.cn, Renmin University of China Hanqing Advanced Institute of Economics and Finance. We thank Jeffrey Coles (associate editor and referee) and Jarrad Harford (the editor) for their helpful suggestions. We also thank Renee Adams, Amir Barnea, David Becher, Alice Bonaimé, Luke DeVault, Ionnis Floros, Vladimir Gatchev, Samir Ghannam, Ivan Gloris, Daniel Greene, Sandy Klasa, Jayanthi Krishnan, Brandon Lockhart, Angie Low, David Mauer, Angela Morgan, Tom Omer, David Reeb, Matthew Serfling, Rick Sias, Johan Sulaeman, Jayanthi Sunder, Rong Wang, Mike Weisbach, Jack Wolf, Ryan Williams, Fei Xie, and Bernard Yeung; seminar participants at Clemson University, Iowa State University, Nanyang Technological University, and National University of Singapore; and conference participants at the 2015 American Accounting Association (AAA) annual meeting, the 2015 Financial Management Association (FMA) annual meeting, the 28th Australasian Finance and Banking Conference (AFBC), and the 2016 China International Conference in Finance (CICF) for useful comments. An earlier version of this paper was titled "Board Effectiveness and Board Connectedness."
} 
their monitoring ability. For example, recent studies explore the social connections of directors, which is referred to as director "connectedness" or "centrality." These studies suggest that well-connected board members have access to superior information and are in higher demand because of their experience or expertise; these attributes benefit the firm in the form of improved monitoring and advising. Consistent with this idea, Larcker, So, and Wang (2013) find that firms with well-connected boards earn higher abnormal returns.

Board connections can also have a dark side, however. Prior work suggests that information spillover from interlocked boards may introduce valuedecreasing management practices such as options backdating (Bizjak, Lemmon, and Whitby (2009), Armstrong and Larcker (2009)). Well-connected directors also may be more concerned about their social status and reputation than about protecting shareholders' interests (Fahlenbrach, Low, and Stulz (2017)). In addition, directors with a large network may be too busy to effectively discipline the CEO (Fich and Shivdasani (2006)) or may be more interested in sitting on the boards of prestigious firms that allow them to enjoy the "quiet life" (Denis, Lee, and Lee (2014)). Therefore, the question of whether and how board connections aid in monitoring and advising is an empirical one.

We fill this gap by examining the effect of director connectedness on their monitoring efficacy and labor market opportunities. We begin by exploring a specific channel through which director connectedness may improve monitoring: the effect of connectedness on financial reporting quality and conservatism. Well-connected directors have better access to information; they also are often more experienced and/or more skilled, and thus can better assess the information. Further, well-connected directors often have more at stake, as they have greater reputations to protect. These factors should lead to stronger incentives to monitor. However, while the entire board plays a central role in monitoring, members of certain committees may be instrumental in specific aspects of monitoring. For example, Coles, Wang, and Zhu (2015) find that the connectedness of nominating committee members is a better predictor of CEO turnover than the connectedness of other board members. Similarly, because audit committee (AC) members are specifically charged with the oversight of financial reporting and thus are particularly concerned about the consequences of fraudulent reports, the connectedness of AC members may be particularly valuable in determining financial reporting quality. For example, connected AC directors are better able to assess fraud risk given their experience, and should enhance monitoring by creating a culture of transparency and accountability, taking whistleblower complaints more seriously, and working more closely with external auditors (PricewaterhouseCoopers (2015)).

We divide directors into three groups based on both their independence and whether they serve on the AC, and calculate the connectedness of each group separately. ${ }^{1}$ Using multiple measures of abnormal accruals, we find that the connectedness of independent, non-co-opted AC members is associated with

\footnotetext{
${ }^{1}$ To ensure true $\mathrm{AC}$ independence, we i) examine outside directors and ii) separate co-opted (i.e., directors who are appointed after the CEO assumes office) from non-co-opted directors. Coles et al. (2014) find that non-co-opted directors are better monitors. Because most ACs are 100\% independent, we use "independent, non-co-opted" directors and "non-co-opted" directors interchangeably
} 
lower earnings management (i.e., higher financial reporting quality) and greater conservatism. In contrast, the connectedness of co-opted AC members and non$\mathrm{AC}$ members has no incremental effect on financial reporting quality. These results are robust to additional tests designed to alleviate endogeneity concerns, including the examination of i) changes around unexpected director deaths, and ii) the effect of AC connectedness in subsamples of firms in which self-selection is less likely to occur, namely, small firms and those with "non-busy" directors. Overall, our results suggest that AC connectedness positively impacts monitoring even after controlling for self-selection.

To further explore the importance of connectedness, we examine the market reaction to the deaths of AC members, which are an exogenous shock to the composition of the AC. Regression results indicate that the market values connected directors; after controlling for other firm and director characteristics, the loss of a highly connected director is associated with a 3\% lower announcement return than the loss of a less connected director. Further, we find that firms with low overall $\mathrm{AC}$ connectedness and those with weaker governance structures experience more negative announcement returns when they lose a highly connected AC member.

Having established that AC connectedness improves financial reporting quality and that the market values connected directors, we next examine the career prospects of AC members after the detection of financial misconduct. Fraudulent reporting can result in lawsuits and U.S. Securities and Exchange Commission (SEC) enforcement actions, which may damage directors' reputations. Previous work shows that directors who sit on the boards of troubled firms face career consequences (Srinivasan (2005), Fich and Shivdasani (2007)), consistent with ex post settling up. In the event of misconduct, well-connected AC members may suffer more severe reputational losses if they are held particularly responsible for the failure. Alternatively, if connected AC members are more valued in the labor market because of their experience and connections, they may be less likely to be penalized than other directors. We initially focus on AC members and find that connectedness improves a director's career prospects, even following misconduct; highly connected AC members are less likely to experience turnover and more likely to obtain future board seats, compared to less connected AC members. Further, although director turnover increases and the number of board seats decreases following misconduct, well-connected AC directors are less likely to suffer these consequences compared to less connected AC directors.

Srinivasan (2005) finds that AC directors are more likely than non-AC directors to experience turnover following the detection of fraud, however. Consequently, we also compare well-connected AC members to well-connected non-AC members, thus holding director connectedness constant and focusing on the impact of financial misconduct and AC membership on career consequences. Consistent with ex post settling up, highly connected AC directors at firms that experience financial misconduct are more likely to experience turnover and less likely to gain future board seats than highly connected non-AC directors.

throughout this article. We include both inside and outside directors in our non-AC connectedness variable, however. 
Overall, the combined results indicate that while connectedness improves the career prospects of $\mathrm{AC}$ directors (even in the event of misconduct), connected $\mathrm{AC}$ directors pay a higher price relative to connected non-AC directors following financial misconduct.

We also examine whether the impact of AC connectedness varies according to the severity of misconduct. Using both cumulative abnormal returns (CARs) around the misconduct revelation date and the settlement amount to proxy for the severity of misconduct, we find that the likelihood of director turnover is higher in cases of severe misconduct. Likewise, directors lose seats on other boards in the 3 years following severe misconduct. However, being highly connected decreases the likelihood of turnover and mitigates the loss of board seats in these cases.

Recent studies suggest a less favorable interpretation for director connectedness, arguing that these directors experience less turnover not because they are good monitors, but due to an "old boys' club" effect (Brown, Gao, Lee, and Stathopoulos (2012), Engelberg, Gao, and Parsons (2013)). Even though we do not definitively prove causality, our empirical evidence is inconsistent with such an interpretation. First, to the extent that this effect stems from the CEO's influence on director selection, our focus on independent, non-co-opted directors is less prone to such conflicts of interest (Barnea and Guedj (2014)). Second, we control for other director-specific characteristics that would be related to an old boys' club effect. Finally, our director death analysis indicates that the market values connected directors; this is inconsistent with the notion that these directors are entrenched or are weak monitors because of their friendship with the CEO.

Our article contributes to the literature on the impact of directors' networks on their monitoring ability. Larcker et al. (2013) find that firms with more central boards earn superior risk-adjusted stock returns. We extend their findings by providing evidence on a specific channel through which board connectedness aids in monitoring: the effect of AC connectedness on financial reporting quality. In a similar vein, Coles et al. (2015) find that the connections of the nominating committee better predict CEO turnover than the connections of other board members and that well-connected directors are able to hire CEO candidates with better skill. They conclude that well-connected directors are better monitors because of their informational advantages and professional expertise. Denis et al. (2014) find that directors with more external connections seek the quiet life by joining boards that are less likely to threaten their time and reputations. Our findings suggest that well-connected directors improve financial reporting quality. Our results also complement other studies that find that industry, legal, or financial expertise of AC members results in reduced earnings management and financial misconduct (Wang, Xie, and Zhu (2015), Krishnan, Wen, and Zhao (2011), and DeFond, Hann, and $\mathrm{Hu}(2005))$.

Our article is also related to Omer, Shelley, and Tice (2016). Whereas their focus is on the likelihood of restatements, we look more broadly at the effect of connectedness on financial reporting quality, as measured by abnormal accruals and accounting conservatism, and on career consequences. Our connectedness measure captures director connections through historical interactions due to prior overlapping directorships, work experiences, social clubs/organizations, and educational experiences. These connections arguably are as strong as, if not stronger 
than, board member connections through contemporaneous board service, which is the scope of director connections in Omer et al. In addition, we examine non-coopted AC members separately from co-opted AC members and non-AC members. Lastly, our results are robust in a sample subject to an exogenous shock (i.e., director deaths) and in other samples designed to alleviate sample selection bias. ${ }^{2}$ Overall, our article fills a gap in the literature by explicitly examining the relations among director connectedness, director career consequences, and financial reporting quality.

Finally, our article is related to the literature on ex post settling up in the director labor market. Previous studies suggest that the director labor market rewards ability and actions that are in shareholders' best interests (Gilson (1990), Harford (2003), and Coles and Hoi (2003)). Bates, Becher, and Wilson (2017) find that directors are disciplined for poor performance but that this relation manifests itself only in the idiosyncratic portion of stock returns. Cashman, Gillan, and Whitby (2010) find that directors with better professional connections, through both common board appointments and overlapping work experience, are less likely to experience ex post settling up if they serve on the board of a firm that restates its financials. Consistent with Harford and Schonlau (2013), who find that experience can counter the normal ex post settling up in the director labor market, we find that connectedness improves the career prospects of AC directors, even following the detection of misconduct. However, relative to connected non-AC directors, connected AC directors experience ex post settling up, in that they are more likely to depart from the firm and less likely to obtain board seats at other firms following the detection of misconduct.

\section{Literature Review and Hypothesis Development}

\section{A. Director Connectedness and Monitoring Effectiveness}

Fama (1980) and Fama and Jensen (1983) posit that a critical function of the board, and specifically outside directors, is to monitor managers to ensure that they act in the best interests of shareholders. However, Hermalin and Weisbach (1998) suggest that a director's willingness to monitor (and potentially replace) the CEO depends on the director's independence. Other studies examine monitoring effectiveness by focusing on the conflicts of interest between managers and independent directors. Ferreira and Laux (2016) find that board independence facilitates monitoring. Shivdasani and Yermack (1999) document that investors react less positively to the appointment of independent directors selected by the CEO, and Hwang and Kim (2009) find that directors who have social connections to the CEO grant higher levels of CEO pay that is unrelated to performance. Cohen, Frazzini, and Malloy (2012) provide evidence that firms appoint independent directors who are overly sympathetic to management; following these appointments, firms significantly increase their earnings management activities and

\footnotetext{
${ }^{2}$ Omer et al. (2016) attempt to control for endogeneity by using average industry connectedness to instrument for firm connectedness. However, Gormley and Matsa (2014) raise concerns about using a group average to instrument for an endogenous variable, as the exclusion restriction is violated whenever an unobserved group-level factor is correlated with the regressor.
} 
CEO compensation. Coles et al. (2014) find that as the fraction of board members appointed after the CEO assumes office (co-option) increases, board monitoring decreases. In sum, these studies suggest that director affiliation with management compromises monitoring diligence.

Director connectedness is distinct from director independence and may also influence monitoring ability. Independent, well-connected directors should have the experience, knowledge, and external connections necessary to effectively monitor and advise top managers. Consistent with this, Wang et al. (2015) find that directors' industry experience improves monitoring effectiveness. Adams and Ferreira (2007) theorize that it is imperative for directors to have sufficient information and knowledge about the firm's operations, and Alam, Chen, Ciccotello, and Ryan (2014) find empirical support for this idea. Further, regulatory reforms enacted as part of the Sarbanes-Oxley Act of 2002 (SOX) call for financial expertise on the AC to ensure that members have sufficient skills to detect financial reporting misconduct (DeFond et al. (2005)). Given the complexity of information disclosed by managers to the board, directors' ability to access external information about market trends, industry developments, and other relevant information is vital in enabling them to analyze and understand this information. Thus, connectedness is a valuable resource that may improve director monitoring effectiveness. ${ }^{3}$

Some studies find evidence that connectedness can compromise monitoring efficacy, however. For example, Bizjak et al. (2009) and Armstrong and Larcker (2009) find that information exchanged through director connections assists in the spread of value-decreasing management practices such as option backdating. Chiu, Teoh, and Tian (2013) find that board interlocks facilitate contagion of earnings management practices. Other studies show that a director's "busyness," defined as the number of board seats held, is negatively associated with monitoring and shareholder wealth. ${ }^{4}$ Therefore, the question of whether better board connections aid in monitoring is an empirical one. The above discussion leads to our first hypothesis in the null form:

Hypothesis 1. All else equal, highly connected, independent, non-co-opted directors are more effective monitors.

\section{B. Director Connectedness, Labor Market Opportunities, and Ex Post Settling Up}

Director connections offer a number of advantages that can increase prestige and reputational capital. For example, well-connected directors have elevated social status that promotes their career prospects (Liu (2014)). Connected directors may sit on more boards and thus feel less dependent on any given board seat and less obligated to any particular CEO (Belliveau, O'Reilly, and Wade (1996)).

\footnotetext{
${ }^{3}$ Alternatively, better connected directors may have greater reputational capital at stake and thus choose to exert more effort compared to less connected directors. In untabulated results, we use AC meeting frequency to proxy for director effort and find that it is not statistically different across lowand high-connected ACs. Thus, our results do not seem to be driven by reputational concerns on the part of better connected committee members.

${ }^{4}$ Although busy directors tend to have more connections, our connectedness measure encompasses more than just the number of connections, so a well-connected director is not necessarily busy. In fact, we find that the correlation between connectedness and busyness is only 0.37 . We control for busyness in our empirical tests, nevertheless.
} 
Better connected directors are respected by other board members, creating a welldefined informational hierarchy that can improve the efficiency of board interactions (He and Huang (2011)). For these reasons, all else equal, well-connected directors should have better career prospects and be less likely to experience turnover.

Hypothesis 2. All else equal, better connected directors have better labor market opportunities.

How well-connected AC directors fare in times of trouble is less clear. According to the notion of ex post settling up, directors should experience higher turnover and/or lose board seats if they take actions that are not in shareholders' best interests. Empirical evidence is mostly consistent with this notion. For example, Gilson (1990) finds that after bankruptcy reorganizations, only $46 \%$ of incumbent directors remain; departing directors hold $33 \%$ fewer other directorships after 3 years. Coles and Hoi (2003) provide evidence that directors of companies that protect themselves against takeovers are less likely to gain additional directorships than directors of companies that reject such provisions. Harford (2003) finds that directors who reject a takeover offer after poor performance obtain fewer future board seats.

However, it is not clear whether the detection of misconduct is an indicator of AC failure. Srinivasan ((2005), p. 299) points out that "because restatements require GAAP violations to be detected, it is not clear whether they represent success or failure by the audit committee." Fich and Shivdasani (2007) and Dyck, Morse, and Zingales (2010) suggest that if directors uncover reporting failures, they may be rewarded with subsequent board appointments. Moreover, unlike CEOs, directors do not directly report to a high authority and thus are difficult to replace (Yermack (2004)).

There is another potential explanation for why connected AC directors may not be subject to as severe labor market consequences as other directors. Harford and Schonlau (2013) posit that while the director labor market should reward ability and punish failure, experience also matters; if experience is valuable enough, it can counter the normal settling up in the labor market. Given that $\mathrm{AC}$ members must be independent and have financial/accounting expertise, good $\mathrm{AC}$ members are hard to find. If well-connected AC members are valued in the labor market because of their scarcity and experience, they may be less likely to be penalized when the firm experiences misconduct. Additionally, the experience obtained while serving on the board of a company that experienced misconduct may be considered valuable. Overall, the effect of financial misconduct on wellconnected AC directors' career consequences is an empirical question.

\section{Data and Variable Definitions}

\section{A. Identifying Director Connectedness}

To capture director connectedness, we use the social network database from BoardEx of Management Diagnostics Ltd., which provides detailed demographic information about the directors and top 5 disclosed earners of publicly traded U.S. companies. BoardEx covers more than 559,667 unique individuals from over 
150,473 unique entities, including private and public companies, universities, and other non-profit organizations, with common employment histories going back as far as 1926. BoardEx covers relational links among directors and other corporate officials through cross-referencing their employment history, educational experience, and professional qualifications/experience. Our sample starts in 2001 and ends in 2010. ${ }^{5}$ We extract data on all executives/directors and their links with all other executives and directors in the database in each year to calculate their social connections. Our definitions of social connections are similar to Liu (2014) and Fracassi (2017). A connection between two individuals is established if they have concurrently worked at the same company (both as executives, both as directors, or one as executive and one as director). We also include their connections via other mechanisms such as civil service in social clubs, charity organizations, and non-profit entities. We count only connections where the date of the information is not missing. Further, we count their connections if they obtain their undergraduate or graduate degrees from the same school and graduate within 2 years of each other. ${ }^{6}$ We calculate connections for all directors in each year, as the cross-reference may change for two reasons. First, newly added individuals may have connections with individuals already in the database. Second, new connections will be established or severed as a result of executives/directors changing firm affiliations. We find that $49 \%$ of independent directors are non-co-opted and therefore "truly" independent (i.e., independent directors who are not appointed by the incumbent CEO), which is comparable to the $47 \%$ reported in Coles et al. (2014). We also calculate connectedness for co-opted directors.

\section{B. Measures of Director Connectedness}

We calculate four common centrality measures developed in network theory, namely degree, betweenness, closeness, and eigenvector centrality. These measures capture not only social ties but, more importantly, the quality of the connections. In the interest of brevity, we further discuss the connectedness

\footnotetext{
${ }^{5}$ BoardEx is commonly used to gauge the social network or connectedness of corporate executives and members of the board of directors (e.g., Fracassi and Tate (2012), Chidambaran, Kedia, and Prabhala (2012), and Cohen et al. (2012)). BoardEx has two potential data biases. First, BoardEx expanded its coverage in 2006 but did not backfill the missing information for new firms (Larcker et al. (2013)). Second, even though BoardEx covers both professional affiliations and other social interactions between executives and directors, it is conceivable that alternative channels exist through which individuals can establish social connections (e.g., marital connections). We address these concerns in robustness tests. For instance, we find similar results when restricting our sample to firms that exist in the database in every year. In addition, to the extent that the bias is related to certain firm characteristics, we use a propensity-score-matched sample and find similar results. Lastly, we limit our sample to start from the post-SOX compliance period (i.e., 2003) and find similar results.

${ }^{6}$ These measures assume that individuals in the network are connected via the shortest paths between them. In reality, people often contact those with whom they share more important experiences. For example, a board member may contact a colleague whom he has known for years, rather than someone with whom he recently shared a charity board experience. It is difficult to agree on a parsimonious measure to rank such connections (e.g., are corporate board connections ranked higher than social boards such as charities, foundations, and academic institutions?). In robustness tests, we examine how long individuals share an experience and assume that the longer a shared experience, i) the stronger the connection and ii) the higher the likelihood that they contact each other to transfer information. We then use the common time between individuals as the weight to rank the connection. Results are robust to using the weighted version of each measure.
} 
measures in the appendices. Appendix A provides detailed explanations of each measure and Appendix B shows how the measures are calculated using a simple network. Summary statistics of the director-level centrality measures are in Panel A of Table B2. Although each centrality measure focuses on a distinct aspect of the individual's importance in the network, the correlation matrix in Panel $\mathrm{B}$ shows that all measures are positively correlated with each other. We use factor analysis to extract the common latent factor that explains the variation across these measures. Panel $\mathrm{C}$ shows that the factor analysis loads on one factor that has an eigenvalue greater than 1 , which explains $63 \%$ of the variation. We use this factor score as our director-level connectedness measure. ${ }^{7}$

Our director-level connectedness measure captures the relative importance of an individual director in the entire social network. Conceptually, it is distinct from director linkage measures that proxy for other director characteristics. However, it is conceivable that our measure may capture some of these other characteristics. For instance, a director with a high level of intelligence or expertise may be able to achieve a higher level of connectedness. Consequently, we explicitly control for director characteristics that may overlap with their connectedness. Specifically, in our multivariate tests we control for i) number of external board seats (BOARD_SEATS); ii) educational background (HIGH_EDU), as prior studies indicate that directors with degrees from prominent institutions are more likely to have a successful career and develop more social connections ${ }^{8}$ iii) number of high-level career appointments that are greater than vice president (HIGH_POST), as career success can reflect intelligence and expertise, which leads to greater reputation (Chemmanur and Paeglis (2005)); and iv) age (D_AGE), which proxies for unobservable characteristics that accumulate with experience.

To reflect the overall connectedness of a firm's directors, we average the individual connectedness values at the board/committee level in each year. We develop three AC/board connectedness measures for our multivariate tests. First, we average the values for all independent, non-co-opted AC directors within each firm-year to obtain AC-level connectedness (AC_CONNECT). Second, we develop a similar measure for co-opted (i.e., non-independent) AC members (AC_OTHR_CONNECT). Finally, we repeat this procedure for non-AC board members (NON_AC_CONNECT). Including all three measures in our tests provides us with a richer understanding of the importance of different groups of directors in monitoring. We examine AC directors separately from other board members because AC members are specifically charged with the oversight of

\footnotetext{
${ }^{7}$ Based on this measure, the most connected directors include Douglas Alexander Warner III (chair of the AC at GE, former CEO of J.P. Morgan Chase), Kevin W. Sharer (director at 3M, former CEO of Amgen, lecturer at Harvard, U.S. Naval Academy graduate), John Fellows Akers (director at New York Times, former CEO of IBM), Tim D. Cook (director at Nike, chief operating officer of Apple, eventual successor to Steve Jobs), and Walter (Jim) James McNerney Jr. (chair at P\&G, CEO of Boeing, CEO of $3 \mathrm{Com})$.

${ }^{8}$ If directors obtained their undergraduate or graduate degree(s) from Brown University, The University of Chicago, Columbia University, Cornell University, Dartmouth College, Duke University, Harvard University, Massachusetts Institute of Technology, Northwestern University, University of Pennsylvania, Princeton University, Stanford University, University of California, Los Angeles, University of California, Berkeley, or Yale University, we classify them as having a degree from a prominent institution.
} 
financial reporting. Our focus on independent, non-co-opted AC directors follows Coles et al. (2014), who find that as the fraction of board members appointed after the CEO assumed office (co-option) increases, board monitoring decreases. ${ }^{9}$

\section{AC, Board, and CEO Characteristics}

We also control for several other AC, board, and CEO characteristics. First, we control for AC members' high position (AC_HIGH_POST), high education (AC_HIGH_EDU), board seats (AC_BOARD_SEATS), and expertise (AC_ACCT EXPERT) by averaging the corresponding director-level measures. Second, we include AC_SIZE, the natural $\log$ of the total number of AC members, and AC_INDEPENDENCE, the number of independent, non-co-opted AC members scaled by total number of AC members. Next, we denote the proportion of busy directors on the AC by AC_BUSY. We define a busy director as an independent, nonco-opted director who serves on 4 or more public firms' boards (Fich and Shivdasani (2006)). We also include an indicator variable (STAGGERED_BOARD) if the board has a staggered election system, as prior studies indicate that staggered boards are associated with poor monitoring and lower firm value (Cohen and Wang (2013), Gompers, Ishii, and Metrick (2003)). Since the ability of the board to monitor depends on the relative power of the CEO versus that of the board (Hermalin and Weisbach (1998), Morse, Nanda, and Seru (2011)), we include CEO connectedness (CEO_CONNECT) as a control for CEO power (El-Khatib, Fogel, and Jandik (2015)). ${ }^{10}$

CEOs may share common experiences even with non-co-opted directors. These connections may enhance communications and information sharing between the CEO and the board, suggesting that the board may be better able to monitor managers (Adams and Ferreira (2007), Raheja (2005), and Engelberg, Gao, and Parsons (2012)). Conversely, recent evidence suggests that these links are problematic and result in higher compensation, lower pay-performance sensitivity, worse firm performance, and reduced likelihood of CEO dismissal (Hwang and Kim (2009), Nguyen (2012), Fracassi and Tate (2012), and Coles et al. (2014)). Further, Chidambaran et al. (2012) and Khanna, Kim, and Lu (2015) find that CEO-director connections have a positive and significant effect on the probability that the firm commits fraud. To control for these connections, we control for both overlapping historical work experience (as directors and/or executives) between the CEO and each independent, non-co-opted AC member, and CEO interlocks with non-co-opted AC members (Fich and Shivdasani (2006)). We then divide the number of independent, non-co-opted AC members who have these connections to the CEO by the total number of independent, non-co-opted AC members (CEO/AC_OVERLAP).

\footnotetext{
${ }^{9}$ Our results are robust to a variety of other measures of connectedness, as outlined in our robustness section.

${ }^{10}$ Alternatively, we use a factor score based on 3 proxies for CEO power: CEO tenure, CEO equity ownership, and CEO/chairman duality. This approach significantly decreases sample size because of CEO-specific data availability, particularly on equity ownership. Regardless, we find similar results in the smaller sample if we include CEO power as a control variable or if we use the 3 proxies separately.
} 


\section{Financial Reporting Quality Measures}

We proxy for financial reporting quality using 3 common accruals-based measures. Extant studies indicate that earnings management occurs via positive abnormal accruals (Healy (1985), Teoh, Welch, and Wong (1998)). Xie (2001) finds that the market overprices discretionary or abnormal accruals, indicating that lower accruals may help mitigate the overpricing problem for investors. High accruals are also associated with internal control weakness disclosures (Doyle, Ge, and McVay (2007)). Thus, lower abnormal accruals are generally associated with improved reporting quality. Our first accruals-based measure (HN) is the absolute value of abnormal accruals based on Hribar and Nichols (2007). The second measure (DGLS) is the error term from the estimation of accruals calculated as the industry-adjusted absolute value of the Dechow and Dichev (2002) residual, based on the cross-sectional adaptation of the model in Dechow, Ge, Larson, and Sloan (2011). Our third measure (AQ) is the Kothari, Leone, and Wasley (2005) performance-matched signed discretionary accruals estimate used by Ashbaugh, LaFond, and Mayhew (2003).

As an additional measure of reporting quality, we examine accounting conservatism. Watts (2003) and Holthausen and Watts (2001) suggest that accounting conservatism has persisted because it alleviates agency problems. We gauge conservatism using 2 common measures: i) the CSCORE measure developed by Khan and Watts (2009), which is based on an augmented Basu (1997) model, and ii) the difference between the skewness of cash flows from operations and the skewness of earnings before extraordinary items (SKEW) (Givoly and Hayn (2000), Beatty, Weber, and Yu (2008)). Detailed descriptions of each measure of reporting quality are in Appendix C.

\section{E. Summary Statistics}

Table 1 presents summary statistics for the variables used in our analysis. All continuous variables are winsorized at the $1 \%$ and $99 \%$ levels and independent variables are lagged 1 year in our multivariate tests. Panel A examines our dependent variables. Independent, non-co-opted AC members experience an average annual turnover rate of $8.9 \%$ in the full sample and $12.7 \%$ in the misconduct sample. On average, directors obtain 1 (2) new board seat(s) over the next 3 years in the full sample (the misconduct sample). The mean (median) reporting quality as measured by HN/DGLS/AQ is comparable to previous studies (Hribar and Nichols (2007), Dechow et al. (2011), and Ashbaugh et al. (2003)). For the measures for accounting conservatism (CSCORE and SKEW), the means and medians are similar to those reported in Khan and Watts (2009) and Beatty et al. (2008).

Panel B of Table 1 examines AC characteristics. Although our connectedness measures have no intuitive explanation because of our factor analysis procedure, the means, medians, and standard deviations are similar, regardless of whether we examine the connectedness of the non-co-opted AC members, co-opted AC members, non-AC members, or CEO. Roughly $9.9 \%$ of the independent nonco-opted directors have social connections/interlock with the CEO. We find that $24.2 \%$ of the independent, non-co-opted $\mathrm{AC}$ members are busy directors. The median $\mathrm{AC}$ has 3 members and the mean AC independence (based on non-co-opted directors) is $63 \%$. Approximately $26 \%$ of AC directors are accounting experts. 
TABLE 1

Descriptive Statistics

Table 1 shows the summary statistics of our variables of interest. Connectedness measures are reported based on their factor scores. Appendix B presents the summary statistics of director-level centrality measures. See Appendices C and D for all variable definitions.

\begin{tabular}{|c|c|c|c|c|c|}
\hline Variable & Mean & Median & Std. Dev. & $\begin{array}{l}\text { Bottom } \\
\text { Quartile }\end{array}$ & $\begin{array}{c}\text { Top } \\
\text { Quartile }\end{array}$ \\
\hline \multicolumn{6}{|l|}{ Panel A. Dependent Variables } \\
\hline D_TURNOVER (full sample) & 0.089 & 0.000 & 0.284 & 0.000 & 0.000 \\
\hline D_TURNOVER (matched sample) & 0.111 & 0.000 & 0.314 & 0.000 & 0.000 \\
\hline D_TURNOVER (misconduct only) & 0.127 & 0.000 & 0.333 & 0.000 & 0.000 \\
\hline FUTURE_BOARDS (full sample) & 1.338 & 1.000 & 3.145 & -1.000 & 3.000 \\
\hline FUTURE_BOARDS (matched sample) & 1.858 & 1.000 & 3.638 & -1.000 & 4.000 \\
\hline FUTURE_BOARDS (misconduct only) & 1.767 & 1.000 & 3.615 & -1.000 & 4.000 \\
\hline $\mathrm{HN}$ & 0.053 & 0.036 & 0.055 & 0.016 & 0.069 \\
\hline DGLS & 0.004 & 0.004 & 0.076 & -0.026 & 0.034 \\
\hline$A Q$ & -0.009 & -0.004 & 0.285 & -0.079 & 0.081 \\
\hline CSCORE & 0.118 & 0.113 & 0.111 & 0.053 & 0.178 \\
\hline SKEW & 0.733 & 0.866 & 0.383 & 0.400 & 1.061 \\
\hline \multicolumn{6}{|l|}{$\underline{\text { Panel B. Audit Committee Variables }}$} \\
\hline AC_CONNECT & -0.011 & -0.309 & 0.941 & -0.670 & 0.324 \\
\hline AC_OTHR_CONNECT & -0.008 & -0.225 & 0.982 & -0.463 & 0.199 \\
\hline NON_AC_CONNECT & 0.002 & -0.093 & 0.990 & -0.466 & 0.267 \\
\hline CEO_CONNECT & -0.000 & -0.257 & 0.998 & -0.372 & 0.084 \\
\hline HIGH_AC_CONNECT & 0.252 & 0.000 & 0.266 & 0.000 & 1.000 \\
\hline CEO/AC_OVVERLAP & 0.099 & 0.079 & 0.166 & 0.000 & 0.172 \\
\hline AC_BUSY & 0.242 & 0.000 & 0.349 & 0.000 & 0.500 \\
\hline AC_SIZE & 3.625 & 3.000 & 0.928 & 3.000 & 4.000 \\
\hline AC_INDEPENDENCE & 0.632 & 0.650 & 0.296 & 0.333 & 1.000 \\
\hline AC_ACCT_EXPERT & 0.257 & 0.250 & 0.239 & 0.000 & 0.333 \\
\hline AC_HIGH_EDU & 0.065 & 0.000 & 0.096 & 0.000 & 0.125 \\
\hline AC_HIGH_POST & 2.858 & 2.000 & 1.975 & 1.000 & 4.000 \\
\hline AC_AGE & 61.640 & 62.000 & 9.276 & 56.000 & 68.000 \\
\hline AC_BOARD_SEATS & 2.584 & 2.000 & 3.220 & 0.000 & 4.000 \\
\hline \multicolumn{6}{|l|}{$\underline{\text { Panel C. Other Controls }}$} \\
\hline FIRM_SIZE (\$millions) & $4,077.700$ & 485.370 & $18,619.120$ & 128.240 & $1,749.109$ \\
\hline $\mathrm{ROA}$ & 0.010 & 0.046 & 0.501 & 0.007 & 0.099 \\
\hline LEVERAGE & 0.175 & 0.104 & 0.247 & 0.004 & 0.270 \\
\hline MTB & 2.619 & 1.864 & 3.841 & 1.182 & 3.101 \\
\hline INST_OWN & 0.484 & 0.525 & 0.344 & 0.136 & 0.801 \\
\hline ANALYSTS & 9.106 & 7.000 & 7.623 & 3.000 & 13.000 \\
\hline STAGGERED_BOARD & 0.381 & 0.000 & 0.283 & 0.000 & 0.000 \\
\hline PRIOR_RET & 0.164 & 0.022 & 0.817 & -0.241 & 0.315 \\
\hline
\end{tabular}

Roughly $6.5 \%$ of non-co-opted $\mathrm{AC}$ members have degrees from prominent schools, and on average they obtain 2.9 high-level positions during their careers. The mean (median) AC director is 61.6 (62) years old and holds 2.6 (2) additional board seats.

Panel $\mathrm{C}$ of Table 1 examines other firm characteristics. Average FIRM_SIZE, measured as total assets, is $\$ 4.1$ billion, with the top (bottom) quartile at $\$ 1,749$ (\$128) million, suggesting a wide coverage of firms in our sample. The mean (median) return on assets (ROA) is 1\% (4.6\%). On average, firms have leverage of $17.5 \%$ and a market-to-book (MTB) ratio of 2.6. Institutional investors own $48 \%$ of the common equity of our sample firms, and an average of 9 analysts follows each firm. Staggered boards are present in $38.1 \%$ of the firm-year observations. The mean (median) stock return over the previous 3 years (PRIOR_RET) is $16.4 \%$ (2.2\%). 


\section{Multivariate Tests}

\section{A. AC Connectedness and Financial Reporting Quality}

In this section, we explore a specific channel through which director connectedness may improve monitoring by examining the effect of connectedness on financial reporting quality. Financial reporting quality is crucial to the firm's information environment and investor risk, and thus can affect both pricing and capital market costs. In the wake of accounting misconduct in the early 2000s, SOX and Regulation Fair Disclosure enacted changes that strengthened the AC and increased its responsibilities and authority. Although recent studies focus solely on the AC (Bédard, Chtourou, and Courteau (2004), Carcello, Neal, Palmrose, and Scholz (2011), and Dhaliwal, Naiker, and Navissi (2010)), our analysis includes the entire board, which allows us to test whether director connectedness outside of the AC also affects reporting quality. Adams and Ferreira (2007) propose that CEOs may be reluctant to share information with independent directors, consistent with the survey results in Hooghiemstra and van Manen (2004), who report that directors express strong doubts about their ability to obtain timely and adequate information from managers. Director connectedness may substitute for such incomplete managerial disclosure by incorporating outside information obtained through the committee's connections. Such information helps directors better understand financial statements and improve corporate governance. Further, connected directors can better assess fraud risks given their experience, and are more concerned about their reputations, so they should be more likely to take steps to minimize the likelihood of non-compliance. Therefore, we propose that better connectedness is associated with improved monitoring and higher quality financial reporting.

We measure financial reporting quality using the accruals-based measures and proxies for conservatism explained in Section III. Our basic regression specification includes control variables used in prior studies (e.g., Dechow, Ge, and Schrand (2010), Givoly and Hayn (2000), and Khan and Watts (2009)). In addition, we calculate connectedness for 3 groups: non-co-opted AC members (AC_CONNECT), co-opted AC members (AC_OTHR_CONNECT), and nonAC members (NON_AC_CONNECT). ${ }^{11}$ We control for AC accounting expertise (AC_ACCT_EXPERT), as prior studies show that it is the SEC's narrowly defined accounting expertise, rather than the broader financial expertise of members, that drives the committee's monitoring effectiveness (DeFond et al. (2005)), Krishnan and Visvanathan (2008)). ${ }^{12}$ We also control for AC size, independence, and busyness to capture the quality of the committee (Anderson, Mansi, and Reeb (2004), Klein (2002), and Bédard et al. (2004)). Finally, we control for 2-digit

\footnotetext{
${ }^{11}$ If a firm has only one type of director on the AC, we set the connectedness measure for the missing type to 0 . However, our results are similar if we instead drop those observations from our sample.

${ }^{12}$ Specifically, we examine the prior work experience and qualifications of each independent, nonco-opted AC member using BoardEx. We classify individuals as accounting experts if they currently hold or have held the position of chief financial officer (CFO), certified public accountant/certified financial analyst (CPA/CFA), controller, comptroller, treasurer, or any other position that is related to financial reporting.
} 
historical Standard Industrial Classification (SIC) industry and year fixed effects when feasible.

Table 2 presents our results. Panel A shows the results using accruals-based measures of reporting quality. Because interpreting economic significance when using the factor score for AC connectedness is difficult, we use a dummy variable equal to 1 if AC_CONNECT is in the top quartile (HIGH_AC_CONNECT). In columns $1-3$, we use ordinary least squares (OLS) to examine the relation between director connectedness and the 3 accruals measures. The coefficient on HIGH_AC_CONNECT is significant in all 3 regressions, suggesting that higher $\mathrm{AC}$ connectedness is associated with lower earnings management (i.e., higher financial reporting quality). Economically, going from low to high AC connectedness is associated with a $32 \%$ to $61 \%$ increase in financial reporting quality, depending on the accruals measure used. ${ }^{13}$ In contrast, we observe insignificant coefficients on AC_OTHR_CONNECT and NON_AC_CONNECT. These findings suggest that the connectedness of independent, non-co-opted AC members is most important in the determination of financial reporting quality; co-opted directors and directors who are not AC members are less likely to be effective monitors. We also find that accounting expertise (AC_ACCT_EXPERT) is positively associated with financial reporting quality, albeit marginally. AC size (AC_SIZE) is positively associated with financial reporting quality, consistent with the notion that larger ACs have more or better "eyes" with which to monitor managers. AC independence (AC_INDEPENDENCE) is unrelated to financial reporting quality, which is not surprising given the $100 \%$ independence requirement for $\mathrm{AC}$ membership in the post-SOX era. AC_BUSY is positive and significant for 2 of the 3 accruals measures, indicating that busy ACs are less effective monitors. The measures for director ability, CEO_CONNECT, and CEO/AC_OVERLAP are not significantly related to audit quality.

To alleviate self-selection concerns, we restrict the sample to include only exogenous shocks to the AC in the form of member deaths. Director deaths are identified by the Audit Analytics Director and Officer Changes database. To quantify the effect of this shock, we calculate the change (year $t+1$ minus year $t-1$ ) in each variable relative to the year of the director death $(t=0)$. Results are provided in columns 4-6 of Panel A of Table 2. For our sample of 122 AC member deaths, the coefficient on the change in $\mathrm{AC}$ connectedness is negative and significant, indicating that increases (decreases) in AC connectedness are associated with improvements (declines) in financial reporting quality. In untabulated results, we find that unlike AC member deaths, the deaths of non-AC members have no significant effect on audit quality. It is conceivable that any change in AC membership might lead to an increase in earnings quality. For example, newly appointed committee members may be more conservative, regardless of whether they are well connected, resulting in an improvement in reporting quality. However, we observe a reduction in AC connectedness in $66 \%$ of our 122 cases of director

\footnotetext{
${ }^{13}$ In untabulated analysis, we use the continuous factor score for AC Connectedness and find similar results. Specifically, we find that a 1-standard-deviation increase in AC Connectedness is associated with a $17.8 \%$ to $31.4 \%$ increase in financial reporting quality. We focus on the dummy variable throughout the paper for ease of interpretation, but our results hold when using the continuous variable.
} 
TABLE 2

\section{Audit Committee Connectedness and Financial Reporting Quality}

Table 2 examines the effect of audit committee $(A C)$ connectedness on financial reporting quality in Panel $A$ and conservatism in Panel B. In Panel A (Panel B), columns 1-3 (1-2) provide full-sample ordinary least squares (OLS) results, and columns 4-6 (3-4) report change regressions based on firms with AC member deaths. All change variables are year $t+1$ minus year $t-1$ values, with year $t$ being the death year. See Appendices $C$ and $D$ for variable definitions. Numbers and variables of interest are in bold. The $t$-statistics (in parentheses) are adjusted for heteroscedasticity using the Huber-White sandwich estimator and are clustered at the firm level. ${ }^{*},{ }^{* *}$, and ${ }^{* *}$ indicate significance at the $10 \%$, $5 \%$, and $1 \%$ levels using 2 -tailed tests, respectively.

Panel A. Accruals-Based Measures Financial Reporting Quality

\begin{tabular}{|c|c|c|c|c|c|c|}
\hline \multirow{4}{*}{ Variable } & \multicolumn{3}{|c|}{ OLS } & \multicolumn{3}{|c|}{ AC Member Deaths (OLS) } \\
\hline & \multicolumn{6}{|c|}{ Dependent Variable } \\
\hline & $\mathrm{HN}$ & DGLS & $A Q$ & $\Delta \mathrm{HN}$ & $\triangle D G L S$ & $\triangle \mathrm{AQ}$ \\
\hline & 1 & 2 & 3 & 4 & 5 & 6 \\
\hline Constant & $\begin{array}{c}0.045 \\
(1.35)\end{array}$ & $\begin{array}{c}0.023 \\
(0.91)\end{array}$ & $\begin{array}{c}0.025 \\
(1.24)\end{array}$ & $\begin{array}{c}0.022 \\
(0.52)\end{array}$ & $\begin{array}{c}0.003 \\
(0.55)\end{array}$ & $\begin{array}{l}-0.004 \\
(-0.33)\end{array}$ \\
\hline HIGH_AC_CONNECT & $\begin{array}{l}-0.032^{* *} \\
(-2.27)\end{array}$ & $\begin{array}{l}-0.002^{\star *} \\
(-2.30)\end{array}$ & $\begin{array}{l}-0.003^{\star *} \\
(-2.41)\end{array}$ & $\begin{array}{l}-0.203^{* *} \\
(-2.50)\end{array}$ & $\begin{array}{l}-0.046^{\star *} \\
(-2.27)\end{array}$ & $\begin{array}{l}-0.072^{* *} \\
(-2.35)\end{array}$ \\
\hline AC_OTHR_CONNECT & $\begin{array}{l}-0.001 \\
(-1.10)\end{array}$ & $\begin{array}{l}-0.000 \\
(-1.02)\end{array}$ & $\begin{array}{r}-0.001 \\
(-1.20)\end{array}$ & $\begin{array}{c}-0.002 \\
(-1.11)\end{array}$ & $\begin{array}{r}-0.007 \\
(-0.83)\end{array}$ & $\begin{array}{l}-0.011 \\
(-0.76)\end{array}$ \\
\hline NON_AC_CONNECT & $\begin{array}{l}-0.001 \\
(-0.78)\end{array}$ & $\begin{array}{l}-0.001 \\
(-0.79)\end{array}$ & $\begin{array}{l}-0.001 \\
(-1.01)\end{array}$ & $\begin{array}{l}-0.001 \\
(-0.35)\end{array}$ & $\begin{array}{l}-0.003 \\
(-0.65)\end{array}$ & $\begin{array}{l}-0.002 \\
(-0.45)\end{array}$ \\
\hline AC_HIGH_POST & $\begin{array}{l}-0.001 \\
(-0.77)\end{array}$ & $\begin{array}{l}-0.000 \\
(-0.75)\end{array}$ & $\begin{array}{l}-0.001 \\
(-0.72)\end{array}$ & $\begin{array}{l}-0.003 \\
(-0.72)\end{array}$ & $\begin{array}{r}-0.007 \\
(-1.20)\end{array}$ & $\begin{array}{l}-0.006 \\
(-0.70)\end{array}$ \\
\hline AC_HIGH_EDU & $\begin{array}{l}-0.001 \\
(-0.56)\end{array}$ & $\begin{array}{l}-0.001 \\
(-0.61)\end{array}$ & $\begin{array}{l}-0.001 \\
(-0.80)\end{array}$ & $\begin{array}{l}-0.004 \\
(-0.62)\end{array}$ & $\begin{array}{c}-0.004 \\
(-0.82)\end{array}$ & $\begin{array}{l}-0.003 \\
(-0.38)\end{array}$ \\
\hline AC_BOARD_SEATS & $\begin{array}{l}-0.003 \\
(-1.30)\end{array}$ & $\begin{array}{l}-0.002 \\
(-1.25)\end{array}$ & $\begin{array}{c}-0.002 \\
(-1.22)\end{array}$ & $\begin{array}{c}-0.007 \\
(-0.89)\end{array}$ & $\begin{array}{c}-0.007 \\
(-1.39)\end{array}$ & $\begin{array}{l}-0.004 \\
(-1.02)\end{array}$ \\
\hline AC_ACCT_EXPERT & $\begin{array}{l}-0.025^{* *} \\
(-2.33)\end{array}$ & $\begin{array}{l}-0.004^{* *} \\
(-2.11)\end{array}$ & $\begin{array}{l}-0.007^{\star} \\
(-1.76)\end{array}$ & $\begin{array}{l}-0.132 \\
(-1.51)\end{array}$ & $\begin{array}{l}-0.032 \\
(-1.55)\end{array}$ & $\begin{array}{l}-0.025 \\
(-1.44)\end{array}$ \\
\hline AC_SIZE & $\begin{array}{l}-0.001 \\
(-0.70)\end{array}$ & $\begin{array}{l}-0.001 \\
(-0.72)\end{array}$ & $\begin{array}{l}-0.001 \\
(-0.59)\end{array}$ & $\begin{array}{l}-0.013 \\
(-1.58)\end{array}$ & $\begin{array}{l}-0.015 \\
(-1.40)\end{array}$ & $\begin{array}{l}-0.011 \\
(-1.46)\end{array}$ \\
\hline AC_INDEPENDENCE & $\begin{array}{c}-0.001 \\
(-1.10)\end{array}$ & $\begin{array}{c}-0.001 \\
(-0.75)\end{array}$ & $\begin{array}{c}-0.007 \\
(-0.63)\end{array}$ & $\begin{array}{c}-0.007 \\
(-0.40)\end{array}$ & $\begin{array}{c}-0.006 \\
(-0.49)\end{array}$ & $\begin{array}{c}-0.006 \\
(-0.55)\end{array}$ \\
\hline AC_BUSY & $\begin{array}{c}0.002^{*} \\
(1.70)\end{array}$ & $\begin{array}{l}0.003^{\star *} \\
(2.02)\end{array}$ & $\begin{array}{c}0.002 \\
(1.54)\end{array}$ & $\begin{array}{c}0.013 \\
(1.45)\end{array}$ & $\begin{array}{c}0.010 \\
(1.25)\end{array}$ & $\begin{array}{c}0.005 \\
(0.90)\end{array}$ \\
\hline CEO_CONNECT & $\begin{array}{c}0.002 \\
(1.50)\end{array}$ & $\begin{array}{c}0.001 \\
(1.13)\end{array}$ & $\begin{array}{r}0.001 \\
(0.76)\end{array}$ & $\begin{array}{c}0.005 \\
(0.77)\end{array}$ & $\begin{array}{c}0.009 \\
(0.52)\end{array}$ & $\begin{array}{c}0.010 \\
(0.86)\end{array}$ \\
\hline CEO/AC_OVERLAP & $\begin{array}{c}0.008 \\
(1.12)\end{array}$ & $\begin{array}{c}0.001 \\
(0.15)\end{array}$ & $\begin{array}{c}0.001 \\
(0.22)\end{array}$ & $\begin{array}{l}-0.003 \\
(-0.82)\end{array}$ & $\begin{array}{c}-0.004 \\
(-0.55)\end{array}$ & $\begin{array}{l}-0.004 \\
(-0.70)\end{array}$ \\
\hline In(FIRM_SIZE) & $\begin{array}{c}0.003 \\
(1.10)\end{array}$ & $\begin{array}{r}0.001 \\
(1.55)\end{array}$ & $\begin{array}{c}0.002 \\
(0.82)\end{array}$ & $\begin{array}{c}0.065^{*} \\
(1.77)\end{array}$ & $\begin{array}{c}0.013^{*} \\
(1.92)\end{array}$ & $\begin{array}{l}0.031^{*} \\
(1.80)\end{array}$ \\
\hline $\mathrm{ROA}$ & $\begin{array}{l}0.020^{*} \\
(1.71)\end{array}$ & $\begin{array}{c}0.017^{*} \\
(1.88)\end{array}$ & $\begin{array}{c}0.052^{*} \\
(1.72)\end{array}$ & $\begin{array}{l}-0.029 \\
(-0.30)\end{array}$ & $\begin{array}{l}-0.027 \\
(-0.89)\end{array}$ & $\begin{array}{l}-0.041 \\
(-0.87)\end{array}$ \\
\hline LEVERAGE & $\begin{array}{c}0.035 \\
(1.25)\end{array}$ & $\begin{array}{r}0.007 \\
(1.25)\end{array}$ & $\begin{array}{r}0.021 \\
(1.50)\end{array}$ & $\begin{array}{c}0.118 \\
(1.15)\end{array}$ & $\begin{array}{c}0.019 \\
(1.20)\end{array}$ & $\begin{array}{l}0.085^{*} \\
(1.82)\end{array}$ \\
\hline INST_OWN & $\begin{array}{l}-0.003^{* *} \\
(-2.02)\end{array}$ & $\begin{array}{l}-0.002 \\
(-1.55)\end{array}$ & $\begin{array}{l}-0.002 \\
(-1.39)\end{array}$ & $\begin{array}{l}-0.118 \\
(-0.82)\end{array}$ & $\begin{array}{l}-0.012 \\
(-1.44)\end{array}$ & $\begin{array}{l}-0.018 \\
(-1.15)\end{array}$ \\
\hline MTB & $\begin{array}{l}-0.001 \\
(-0.80)\end{array}$ & $\begin{array}{l}-0.001 \\
(-1.11)\end{array}$ & $\begin{array}{l}-0.001 \\
(-0.93)\end{array}$ & $\begin{array}{l}-0.022 \\
(-1.33)\end{array}$ & $\begin{array}{l}-0.002 \\
(-0.93)\end{array}$ & $\begin{array}{l}-0.006^{*} \\
(-1.75)\end{array}$ \\
\hline ANALYSTS & $\begin{array}{l}-0.001^{* * *} \\
(-2.85)\end{array}$ & $\begin{array}{l}-0.001^{* * *} \\
(-2.70)\end{array}$ & $\begin{array}{l}-0.001^{\star \star *} \\
(-2.92)\end{array}$ & $\begin{array}{l}-0.011 \\
(-1.20)\end{array}$ & $\begin{array}{l}-0.003 \\
(-1.38)\end{array}$ & $\begin{array}{l}-0.003 \\
(-0.92)\end{array}$ \\
\hline LIT_RISK & $\begin{array}{l}0.006^{* *} \\
(2.13)\end{array}$ & $\begin{array}{l}0.002^{*} \\
(1.82)\end{array}$ & $\begin{array}{l}0.008^{* *} \\
(2.22)\end{array}$ & $\begin{array}{l}-0.051 \\
(-1.32)\end{array}$ & $\begin{array}{l}-0.003 \\
(-0.75)\end{array}$ & $\begin{array}{l}-0.003 \\
(-0.30)\end{array}$ \\
\hline$\sigma(\mathrm{SALES})$ & $\begin{array}{l}0.031^{* *} \\
(2.33)\end{array}$ & $\begin{array}{l}0.003^{*} \\
(1.90)\end{array}$ & $\begin{array}{l}0.008^{*} \\
(1.91)\end{array}$ & $\begin{array}{l}0.205^{*} \\
(1.78)\end{array}$ & $\begin{array}{l}0.043^{*} \\
(1.88)\end{array}$ & $\begin{array}{l}0.112^{*} \\
(1.85)\end{array}$ \\
\hline$\sigma(\mathrm{OCF})$ & $\begin{array}{l}0.031^{\star * \star} \\
(3.23)\end{array}$ & $\begin{array}{l}0.003^{*} \\
(1.77)\end{array}$ & $\begin{array}{l}0.008^{\star *} \\
(2.22)\end{array}$ & $\begin{array}{l}1.103^{*} \\
(1.92)\end{array}$ & $\begin{array}{l}0.570^{* *} \\
(2.33)\end{array}$ & $\begin{array}{l}0.160^{*} \\
(1.74)\end{array}$ \\
\hline OPER_CYCLE & $\begin{array}{l}0.009^{*} \\
(1.91)\end{array}$ & $\begin{array}{l}0.002^{*} \\
(1.88)\end{array}$ & $\begin{array}{l}0.003^{*} \\
(1.77)\end{array}$ & $\begin{array}{l}0.327^{*} \\
(1.69)\end{array}$ & $\begin{array}{l}0.119^{* *} \\
(2.35)\end{array}$ & $\begin{array}{l}0.206^{\star * *} \\
(3.19)\end{array}$ \\
\hline Industry and year dummies & Yes & Yes & Yes & No & No & No \\
\hline $\begin{array}{l}\text { No. of obs. } \\
\text { Adj. } R^{2}\end{array}$ & $\begin{array}{r}20,421 \\
0.155\end{array}$ & $\begin{array}{r}20,421 \\
0.118\end{array}$ & $\begin{array}{r}19,345 \\
0.122\end{array}$ & $\begin{array}{r}122 \\
0.416\end{array}$ & $\begin{array}{r}122 \\
0.245\end{array}$ & $\begin{array}{r}122 \\
0.471\end{array}$ \\
\hline & & & & & (continue & next page) \\
\hline
\end{tabular}


TABLE 2 (continued)

Audit Committee Connectedness and Financial Reporting Quality

Panel B. Conservatism

\begin{tabular}{|c|c|c|c|c|}
\hline \multirow{4}{*}{ Variable } & \multicolumn{2}{|c|}{ OLS } & \multicolumn{2}{|c|}{ AC Member Deaths (OLS) } \\
\hline & \multicolumn{4}{|c|}{ Dependent Variable } \\
\hline & CSCORE & SKEW & $\triangle \mathrm{CSCORE}$ & $\triangle$ SKEW \\
\hline & 1 & 2 & 3 & 4 \\
\hline Constant & $\begin{array}{l}0.186^{\star * *} \\
(16.34)\end{array}$ & $\begin{array}{l}-0.552^{\star \star \star *} \\
(-23.90)\end{array}$ & $\begin{array}{l}0.050^{*} \\
(1.90)\end{array}$ & $\begin{array}{l}0.066^{*} \\
(1.93)\end{array}$ \\
\hline HIGH_AC_CONNECT & $\begin{array}{l}0.021^{* * *} \\
(2.78)\end{array}$ & $\begin{array}{l}0.167^{* * *} \\
(3.19)\end{array}$ & $\begin{array}{l}0.172^{* *} \\
(2.45)\end{array}$ & $\begin{array}{l}0.300^{* *} \\
(2.51)\end{array}$ \\
\hline AC_OTHR_CONNECT & $\begin{array}{c}0.004 \\
(1.20)\end{array}$ & $\begin{array}{c}0.013 \\
(1.30)\end{array}$ & $\begin{array}{c}0.002 \\
(0.35)\end{array}$ & $\begin{array}{r}0.007 \\
(0.45)\end{array}$ \\
\hline NON_AC_CONNECT & $\begin{array}{c}0.003 \\
(1.25)\end{array}$ & $\begin{array}{l}0.018 \\
(1.15)\end{array}$ & $\begin{array}{c}0.008 \\
(1.25)\end{array}$ & $\begin{array}{c}0.011 \\
(0.73)\end{array}$ \\
\hline AC_HIGH_POST & $\begin{array}{c}0.002 \\
(0.91)\end{array}$ & $\begin{array}{c}0.012 \\
(0.82)\end{array}$ & $\begin{array}{c}0.003 \\
(0.30)\end{array}$ & $\begin{array}{l}0.008 \\
(0.55)\end{array}$ \\
\hline AC_HIGH_EDU & $\begin{array}{c}0.002 \\
(0.61)\end{array}$ & $\begin{array}{c}0.010 \\
(0.80)\end{array}$ & $\begin{array}{l}0.003 \\
(0.73)\end{array}$ & $\begin{array}{c}0.002 \\
(0.31)\end{array}$ \\
\hline AC_BOARD_SEATS & $\begin{array}{c}0.003 \\
(0.66)\end{array}$ & $\begin{array}{c}0.006 \\
(0.42)\end{array}$ & $\begin{array}{c}0.003 \\
(0.35)\end{array}$ & $\begin{array}{c}0.004 \\
(0.47)\end{array}$ \\
\hline AC_ACCT_EXPERT & $\begin{array}{c}0.005 \\
(0.89)\end{array}$ & $\begin{array}{l}0.006^{*} \\
(1.93)\end{array}$ & $\begin{array}{c}0.056 \\
(1.05)\end{array}$ & $\begin{array}{c}0.279 \\
(1.03)\end{array}$ \\
\hline AC_SIZE & $\begin{array}{c}0.002 \\
(1.09)\end{array}$ & $\begin{array}{c}0.002 \\
(0.85)\end{array}$ & $\begin{array}{l}0.018^{*} \\
(1.72)\end{array}$ & $\begin{array}{l}0.031^{*} \\
(1.90)\end{array}$ \\
\hline AC_INDEPENDENCE & $\begin{array}{r}0.001 \\
(0.77)\end{array}$ & $\begin{array}{c}0.002 \\
(0.66)\end{array}$ & $\begin{array}{c}0.010 \\
(1.03)\end{array}$ & $\begin{array}{c}0.022 \\
(1.21)\end{array}$ \\
\hline AC_BUSY & $\begin{array}{l}-0.010^{* *} \\
(-2.30)\end{array}$ & $\begin{array}{l}-0.018^{\text {***}} \\
(-2.70)\end{array}$ & $\begin{array}{l}-0.010 \\
(-1.08)\end{array}$ & $\begin{array}{l}-0.020 \\
(-1.15)\end{array}$ \\
\hline CEO_CONNECT & $\begin{array}{l}-0.001 \\
(-1.43)\end{array}$ & $\begin{array}{l}-0.001 \\
(-0.79)\end{array}$ & $\begin{array}{l}-0.011 \\
(-1.16)\end{array}$ & $\begin{array}{l}-0.062 \\
(-0.83)\end{array}$ \\
\hline CEO/AC_OVERLAP & $\begin{array}{l}-0.008 \\
(-0.99)\end{array}$ & $\begin{array}{l}-0.002 \\
(-0.59)\end{array}$ & $\begin{array}{l}-0.030 \\
(-0.92)\end{array}$ & $\begin{array}{l}-0.367 \\
(-1.43)\end{array}$ \\
\hline In(FIRM_SIZE) & $\begin{array}{l}-0.027^{* * *} \\
(-22.67)\end{array}$ & $\begin{array}{l}-0.016^{* \star} \\
(-2.32)\end{array}$ & $\begin{array}{l}-0.027^{* *} \\
(-1.99)\end{array}$ & $\begin{array}{l}-0.195^{* * \star} \\
(-2.92)\end{array}$ \\
\hline $\mathrm{ROA}$ & $\begin{array}{l}-0.015^{\star *} \\
(-2.22)\end{array}$ & $\begin{array}{l}-0.033^{\star \star} \\
(-2.52)\end{array}$ & $\begin{array}{l}-0.023^{*} \\
(-1.70)\end{array}$ & $\begin{array}{l}-0.441^{\text {** }} \\
(-2.36)\end{array}$ \\
\hline LEVERAGE & $\begin{array}{l}0.032^{* * *} \\
(3.89)\end{array}$ & $\begin{array}{l}0.026^{\star *} \\
(2.50)\end{array}$ & $\begin{array}{c}0.055 \\
(1.11)\end{array}$ & $\begin{array}{l}0.507^{\star \star} \\
(2.01)\end{array}$ \\
\hline INST_OWN & $\begin{array}{l}0.018^{\text {***}} \\
(3.01)\end{array}$ & $\begin{array}{l}0.016^{\text {***}} \\
(2.60)\end{array}$ & $\begin{array}{c}-0.116 \\
(-1.35)\end{array}$ & $\begin{array}{l}-0.060 \\
(-1.46)\end{array}$ \\
\hline MTB & $\begin{array}{l}-0.004^{\star * *} \\
(-13.33)\end{array}$ & $\begin{array}{l}-0.001^{\text {***}} \\
(-3.45)\end{array}$ & $\begin{array}{l}-0.004^{* *} \\
(-2.30)\end{array}$ & $\begin{array}{l}-0.014 \\
(-1.30)\end{array}$ \\
\hline ANALYSTS & $\begin{array}{l}-0.001^{* * *} \\
(-2.85)\end{array}$ & $\begin{array}{l}-0.001^{* *} \\
(-2.16)\end{array}$ & $\begin{array}{l}-0.006^{*} \\
(-1.85)\end{array}$ & $\begin{array}{r}-0.017 \\
(-1.00)\end{array}$ \\
\hline LIT_RISK & $\begin{array}{l}-0.007^{\star * *} \\
(-9.67)\end{array}$ & $\begin{array}{l}-0.003^{\star \star *} \\
(-3.05)\end{array}$ & $\begin{array}{l}-0.002 \\
(-0.30)\end{array}$ & $\begin{array}{l}-0.022 \\
(-0.90)\end{array}$ \\
\hline$\sigma$ (SALES) & $\begin{array}{l}-0.016^{* *} \\
(-2.20)\end{array}$ & 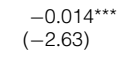 & $\begin{array}{l}-0.355^{* * *} \\
(-4.02)\end{array}$ & $\begin{array}{l}-0.511^{*} \\
(-1.92)\end{array}$ \\
\hline$\sigma$ (OCF) & 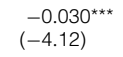 & $\begin{array}{l}-0.033^{\text {*** }} \\
(-3.16)\end{array}$ & $\begin{array}{l}-0.319^{* *} \\
(-2.02)\end{array}$ & $\begin{array}{l}-0.085^{* *} \\
(-2.10)\end{array}$ \\
\hline OPER_CYCLE & $\begin{array}{l}-0.005^{\star * *} \\
(-2.73)\end{array}$ &  & $\begin{array}{l}-0.032^{* *} \\
(-2.20)\end{array}$ & $\begin{array}{l}-0.031^{*} \\
(-1.85)\end{array}$ \\
\hline Industry and year dummies & Yes & Yes & No & No \\
\hline $\begin{array}{l}\text { No. of obs. } \\
\text { Adj. } R^{2}\end{array}$ & $\begin{array}{r}20,421 \\
0.360\end{array}$ & $\begin{array}{r}20,421 \\
0.163 \\
\end{array}$ & $\begin{array}{r}122 \\
0.265\end{array}$ & $\begin{array}{r}122 \\
0.310\end{array}$ \\
\hline
\end{tabular}

deaths, which is inconsistent with this notion. We also run regressions similar to those in columns 4-6 of Panel A, but on our entire sample. Untabulated results show that the coefficient on the change in HIGH_AC_CONNECT is negative, but not statistically significant, possibly because of the lack of variation in the change 
in HIGH_AC_CONNECT due to the persistence of board composition over time. Given these results, it is unlikely that our findings are driven by a change in the structure or composition of the AC.

In Panel B of Table 2, we examine the effect of connectedness on accounting conservatism. All specifications suggest that HIGH_AC_CONNECT is positively associated with conservatism. More specifically, the results in columns 1 and 2 indicate that going from low to high AC connectedness is associated with a $17.8 \%(22.8 \%)$ increase in conservatism using the CSCORE (SKEW) measure, respectively. As in Panel A, busy ACs are less effective monitors. In columns 3 and 4, we again use AC member deaths for identification and find that the change in $\mathrm{AC}$ connectedness after the exogenous shock is positively related to the change in CSCORE and SKEW. Overall, Table 2 shows that director connectedness positively impacts financial reporting quality and conservatism, which supports Hypothesis 1. This effect is strongest for non-co-opted members of the AC.

\section{B. Further Check on Self-Selection Bias}

The previous section uses instances of director death to establish identification. We further address the issue of self-selection between directors and firms by partitioning on characteristics that affect the ability of directors to self-select. If well-connected directors are self-selecting, they are more likely to choose to sit on larger rather than smaller firms, as larger firms are more prestigious and provide higher director compensation. Knyazeva, Knyazeva, and Masulis (2013) suggest that more qualified directors tend to join larger firms that offer more visibility and greater reputational benefits. Moreover, large firms receive scrutiny from many directions, including analysts and auditors, which could result in better financial reporting and more conservative accounting. In contrast, directors in small firms arguably have fewer external directorship opportunities and thus are less likely to self-select into high-quality firms. Consequently, our hypothesized negative relation between financial reporting quality and AC connectedness should be more pronounced in large firms than in small firms. To examine this, in Panel A of Table 3 we revisit our analysis on financial reporting quality by separating our sample into large (columns 1-3) and small (columns 4-6) firms, where we define large firms as those being in the top quartile based on size. ${ }^{14}$ Results show that the effect of high AC connectedness is significant for small firms but not for large firms, suggesting that the relation between AC connectedness and financial reporting quality is not driven by connected directors self-selecting into higher quality firms. ${ }^{15}$ The lack of a significant relation in the large firms is perhaps a bit surprising and indicates that better connected AC members have little incremental effect on financial reporting quality in large firms that

\footnotetext{
${ }^{14}$ Small firms include the bottom 3 quartiles. Similar results remain if we use only the bottom quartile.

${ }^{15}$ Chow-tests show that the differences in HIGH_AC_CONNECT coefficients between large and small firms are significant at the $5 \%$ level for HN, insignificant for DGLS, and significant at the $10 \%$ level for AQ.
} 
are already highly scrutinized, whereas their influence in smaller, less scrutinized firms is stronger and thus is more likely to prevent earnings management. ${ }^{16}$

We also split our sample into busy and non-busy ACs; a busy AC is one in which more than half of the members sit on 4 or more boards. Busy directors are likely in greater demand and thus have more choices in the labor market than less busy directors. Less busy directors should have fewer directorship opportunities and thus be less likely to self-select into high-quality firms. Consistent with the results in Panel A, Panel B of Table 3 shows that the effect of AC connectedness on reporting quality is significant only in non-busy ACs. ${ }^{17}$

In untabulated results, we find that the effect of $\mathrm{AC}$ connectedness is significantly larger in firms with low institutional ownership. To the extent that institutional ownership is associated with more diligent corporate governance, these findings provide further evidence that the relation between connectedness and reporting quality is not driven by well-connected directors self-selecting into highquality firms.

\section{Market Reaction to the Death of an AC Member}

Thus far we establish that well-connected ACs are better monitors and are associated with high financial reporting quality, consistent with the notion that the $\mathrm{AC}$ is instrumental in financial reporting quality and integrity. In this section, we provide further evidence on the value of connectedness by examining the market reaction to news of an exogenous shock to the structure of the board, in particular, the death of an AC member. The CAR is calculated as the raw daily return minus the value-weighted market return over a $(-2,+2)$ window surrounding the announcement of the death; our results are robust to the use of other windows. The mean (median) CAR for the whole sample is $-1.4 \%(-0.4 \%)$. The average CAR for high- (low-) connectedness directors is $-3.1 \%(-0.8 \%)$, and a $t$-test shows that the difference is statistically significant.

In Table 4, we compare the reaction to the loss of high- versus lowconnectedness directors, controlling for other director and firm characteristics. In column 1, we find that the dummy variable HIGH_D_CONNECT (indicating that director connectedness is in the top quartile) is negative and significant, suggesting that the loss of a director with a high level of connectedness is associated with a $3 \%$ lower announcement return than the loss of a less connected director. To examine whether the death of a highly connected director is particularly harmful in an AC with low overall connectedness, we interact HIGH_D_CONNECT with LOW_AC_CONNECT, defined as the AC itself being in the bottom quartile of connectedness in the year before the director's death. Column 2 shows that HIGH_D_CONNECT remains negative and significant, and the interaction term is also negative and significant, suggesting that the loss of a highly connected director in an $\mathrm{AC}$ with low connectedness generates an even more negative

\footnotetext{
${ }^{16}$ We find that all else equal, large firms have higher financial reporting quality than small firms, consistent with the notion that large firms face more scrutiny and directors have relatively less impact on financial reporting quality.

${ }^{17}$ Chow-tests confirm that the differences in HIGH_AC_CONNECT coefficients between busy and non-busy AC samples are significant. Specifically, differences are significant at the $5 \%$ for $\mathrm{HN}$, at the $1 \%$ level for DGLS, and at the $5 \%$ level for AQ.
} 


\section{Firm Size, Director Busyness, and Director Connectedness}

Table 3 examines the effect of audit committee (AC) connectedness on financial reporting quality by separating the sample into large and small firms and boards with busy and non-busy AC members. Large firms are those that are in the top quartile of firm size as measured by total assets and small firms are those that are in the bottom 3 quartiles. AC_BUSY identifies busy ACs, which are defined as firms with ACs where more than half of the members sit on more than 4 boards. Control variables include the other variables in Table 2. See Appendices $C$ and $D$ for other variable definitions. Numbers and variables of interest are in bold. The $t$-statistics (in parentheses) are adjusted for heteroscedasticity using the HuberWhite sandwich estimator and are corrected for clustering at the firm level. * ${ }^{* *}$, and ${ }^{* * *}$ indicate significance at the $10 \%$, $5 \%$, and $1 \%$ levels using 2 -tailed tests, respectively.

Panel A. Firm Size

\begin{tabular}{|c|c|c|c|c|c|c|}
\hline \multirow{4}{*}{ Variable } & \multicolumn{3}{|c|}{ Large Firms } & \multicolumn{3}{|c|}{ Small Firms } \\
\hline & \multicolumn{6}{|c|}{ Dependent Variable } \\
\hline & $\mathrm{HN}$ & DGLS & $A Q$ & $\mathrm{HN}$ & DGLS & $A Q$ \\
\hline & 1 & 2 & 3 & 4 & 5 & 6 \\
\hline Constant & $\begin{array}{c}0.013 \\
(1.02)\end{array}$ & $\begin{array}{l}0.026 \\
(1.09)\end{array}$ & $\begin{array}{l}0.022 \\
(0.99)\end{array}$ & $\begin{array}{c}0.092 \\
(1.60)\end{array}$ & $\begin{array}{c}0.010 \\
(1.15)\end{array}$ & $\begin{array}{r}0.031 \\
(0.88)\end{array}$ \\
\hline HIGH_AC_CONNECT & $\begin{array}{l}-0.001 \\
(-0.89)\end{array}$ & $\begin{array}{l}-0.001 \\
(-1.29)\end{array}$ & $\begin{array}{l}-0.002 \\
(-1.26)\end{array}$ & $\begin{array}{l}-0.004^{\star \star \star} \\
(-2.79)\end{array}$ & $\begin{array}{l}-0.001^{\star \star} \\
(-2.09)\end{array}$ & $\begin{array}{l}-0.006^{\star \star} \\
(-2.31)\end{array}$ \\
\hline AC_OTHR_CONNECT & $\begin{array}{l}-0.001 \\
(-0.39)\end{array}$ & $\begin{array}{l}-0.001 \\
(-0.51)\end{array}$ & $\begin{array}{l}-0.000 \\
(-0.36)\end{array}$ & $\begin{array}{l}-0.001 \\
(-0.92)\end{array}$ & $\begin{array}{l}-0.001 \\
(-0.82)\end{array}$ & $\begin{array}{l}-0.001 \\
(-0.61)\end{array}$ \\
\hline NON_AC_CONNECT & $\begin{array}{l}-0.001 \\
(-0.36)\end{array}$ & $\begin{array}{l}-0.001 \\
(-0.29)\end{array}$ & $\begin{array}{l}-0.001 \\
(-0.40)\end{array}$ & $\begin{array}{l}-0.003 \\
(-0.87)\end{array}$ & $\begin{array}{l}-0.003 \\
(-0.90)\end{array}$ & $\begin{array}{l}-0.003 \\
(-0.99)\end{array}$ \\
\hline AC_ACCT_EXPERT & $\begin{array}{l}-0.025 \\
(-1.42)\end{array}$ & $\begin{array}{l}-0.004 \\
(-1.42)\end{array}$ & $\begin{array}{l}-0.005 \\
(-1.59)\end{array}$ & $\begin{array}{l}-0.020^{*} \\
(-1.86)\end{array}$ & $\begin{array}{l}-0.002^{*} \\
(-1.89)\end{array}$ & $\begin{array}{l}-0.006^{\star} \\
(-1.83)\end{array}$ \\
\hline CEO_CONNECT & $\begin{array}{l}0.000 \\
(0.27)\end{array}$ & $\begin{array}{r}0.001 \\
(0.34)\end{array}$ & $\begin{array}{r}0.001 \\
(0.50)\end{array}$ & $\begin{array}{r}0.001 \\
(0.50)\end{array}$ & $\begin{array}{r}0.001 \\
(0.69)\end{array}$ & $\begin{array}{r}0.001 \\
(0.86)\end{array}$ \\
\hline CEO/AC_OVERLAP & $\begin{array}{r}0.007 \\
(0.96)\end{array}$ & $\begin{array}{l}0.002 \\
(0.91)\end{array}$ & $\begin{array}{l}0.002 \\
(0.90)\end{array}$ & $\begin{array}{l}0.008 \\
(0.55)\end{array}$ & $\begin{array}{r}0.001 \\
(0.60)\end{array}$ & $\begin{array}{r}0.001 \\
(0.31)\end{array}$ \\
\hline $\begin{array}{l}\text { Controls } \\
\text { Industry and year dummies }\end{array}$ & $\begin{array}{l}\text { Yes } \\
\text { Yes }\end{array}$ & $\begin{array}{l}\text { Yes } \\
\text { Yes }\end{array}$ & $\begin{array}{l}\text { Yes } \\
\text { Yes }\end{array}$ & $\begin{array}{l}\text { Yes } \\
\text { Yes }\end{array}$ & $\begin{array}{l}\text { Yes } \\
\text { Yes }\end{array}$ & $\begin{array}{l}\text { Yes } \\
\text { Yes }\end{array}$ \\
\hline $\begin{array}{l}\text { No. of obs. } \\
\text { Adj. } R^{2}\end{array}$ & $\begin{array}{l}5,105 \\
0.167\end{array}$ & $\begin{array}{l}5,105 \\
0.122\end{array}$ & $\begin{array}{l}4,836 \\
0.137\end{array}$ & $\begin{array}{r}15,316 \\
0.140\end{array}$ & $\begin{array}{r}15,316 \\
0.106\end{array}$ & $\begin{array}{r}14,509 \\
0.110\end{array}$ \\
\hline \multicolumn{7}{|l|}{ Panel B. Director Busyness } \\
\hline & \multicolumn{3}{|c|}{ Busy AC } & \multicolumn{3}{|c|}{ Non-Busy AC } \\
\hline & \multicolumn{6}{|c|}{ Dependent Variable } \\
\hline & $\mathrm{HN}$ & DGLS & $A Q$ & $\mathrm{HN}$ & DGLS & $A Q$ \\
\hline Variable & 1 & 2 & 3 & 4 & 5 & 6 \\
\hline Constant & $\begin{array}{l}0.050 \\
(0.66)\end{array}$ & $\begin{array}{r}0.007 \\
(0.43)\end{array}$ & $\begin{array}{l}0.031 \\
(0.77)\end{array}$ & $\begin{array}{l}0.073 \\
(1.23)\end{array}$ & $\begin{array}{l}0.015 \\
(1.12)\end{array}$ & $\begin{array}{l}0.015 \\
(0.87)\end{array}$ \\
\hline HIGH_AC_CONNECT & $\begin{array}{l}-0.001 \\
(-0.81)\end{array}$ & $\begin{array}{l}-0.000 \\
(-1.18)\end{array}$ & $\begin{array}{l}-0.001 \\
(-1.22)\end{array}$ & $\begin{array}{l}-0.004^{\star \star \star} \\
(-2.60)\end{array}$ & $\begin{array}{l}-0.001^{\star \star} \\
(-2.52)\end{array}$ & $\begin{array}{l}-0.005^{\star \star} \\
(-2.41)\end{array}$ \\
\hline AC_OTHR_CONNECT & $\begin{array}{l}-0.001 \\
(-0.39)\end{array}$ & $\begin{array}{l}-0.000 \\
(-0.45)\end{array}$ & $\begin{array}{l}-0.001 \\
(-0.79)\end{array}$ & $\begin{array}{c}-0.002 \\
(-1.49)\end{array}$ & $\begin{array}{l}-0.002 \\
(-1.23)\end{array}$ & $\begin{array}{l}-0.002 \\
(-1.41)\end{array}$ \\
\hline NON_AC_CONNECT & $\begin{array}{l}-0.001 \\
(-0.78)\end{array}$ & $\begin{array}{l}-0.001 \\
(-0.90)\end{array}$ & $\begin{array}{l}-0.001 \\
(-0.70)\end{array}$ & $\begin{array}{l}-0.002 \\
(-0.93)\end{array}$ & $\begin{array}{l}-0.002 \\
(-0.89)\end{array}$ & $\begin{array}{l}-0.002 \\
(-0.93)\end{array}$ \\
\hline AC_ACCT_EXPERT & $\begin{array}{l}-0.022^{\star \star} \\
(-2.20)\end{array}$ & $\begin{array}{l}-0.004^{*} \\
(-1.85)\end{array}$ & $\begin{array}{l}-0.008^{\star} \\
(-1.79)\end{array}$ & $\begin{array}{l}-0.011^{\star} \\
(-1.70)\end{array}$ & $\begin{array}{l}-0.001^{\star} \\
(-1.68)\end{array}$ & $\begin{array}{l}-0.004^{\star \star} \\
(-2.03)\end{array}$ \\
\hline CEO_CONNECT & $\begin{array}{r}0.001 \\
(1.30)\end{array}$ & $\begin{array}{r}0.001 \\
(0.95)\end{array}$ & $\begin{array}{r}0.001 \\
(1.10)\end{array}$ & $\begin{array}{r}0.001 \\
(0.88)\end{array}$ & $\begin{array}{l}0.000 \\
(1.17)\end{array}$ & $\begin{array}{r}0.000 \\
(1.40)\end{array}$ \\
\hline CEO/AC_OVERLAP & $\begin{array}{l}0.005 \\
(0.31)\end{array}$ & $\begin{array}{c}0.001 \\
(0.24)\end{array}$ & $\begin{array}{c}0.002 \\
(0.49)\end{array}$ & $\begin{array}{l}0.008 \\
(1.40)\end{array}$ & $\begin{array}{r}0.001 \\
(1.31)\end{array}$ & $\begin{array}{r}0.001 \\
(1.33)\end{array}$ \\
\hline $\begin{array}{l}\text { Controls } \\
\text { Industry and year dummies }\end{array}$ & $\begin{array}{l}\text { Yes } \\
\text { Yes }\end{array}$ & $\begin{array}{l}\text { Yes } \\
\text { Yes }\end{array}$ & $\begin{array}{l}\text { Yes } \\
\text { Yes }\end{array}$ & $\begin{array}{l}\text { Yes } \\
\text { Yes }\end{array}$ & $\begin{array}{l}\text { Yes } \\
\text { Yes }\end{array}$ & $\begin{array}{l}\text { Yes } \\
\text { Yes }\end{array}$ \\
\hline $\begin{array}{l}\text { No. of obs. } \\
\text { Adj. } R^{2}\end{array}$ & $\begin{array}{l}5,289 \\
0.188\end{array}$ & $\begin{array}{l}5,289 \\
0.156\end{array}$ & $\begin{array}{l}5,010 \\
0.177\end{array}$ & $\begin{array}{r}15,132 \\
0.099\end{array}$ & $\begin{array}{r}15,132 \\
0.088\end{array}$ & $\begin{array}{r}14,335 \\
0.085\end{array}$ \\
\hline
\end{tabular}


market reaction. Similarly, in column 3 we interact HIGH_D_CONNECT with a dummy variable that indicates the level of corporate governance. In particular, POOR_GOVERN is a dummy variable that indicates the firm is in the top quartile in terms of anti-takeover clauses according to the Bebchuk, Cohen, and Ferrell (2009) E-index. ${ }^{18}$ HIGH_D_CONNECT is negative and significant. The interaction term between connectedness and poor governance is also negative and significant, suggesting that in a poor-governance environment, the negative effect of the loss of a highly connected director is more pronounced. In our tests we control for a number of director-specific factors, including age, gender, tenure, and board seats held before their death. In addition, we include a dummy variable for accounting expertise, isolating the director connectedness effect from professional expertise. Overall, our evidence supports the notion that director connectedness is valued by investors, consistent with Hypothesis $1 .^{19}$

\section{Director Connectedness and Career Consequences}

Having established that AC connectedness improves financial reporting quality and that the market values connected directors, we next examine whether connected directors have better labor market opportunities. We use cases of detected misconduct as the platform to test this idea. In particular, we use the Audit Analytics Non-Compliance database to identify earnings restatements, and the Legal Case database to identify legal actions related to accounting malpractice, financial reporting, fraud, or reported Accounting and Auditing Enforcement Release (AAER) suits; our results are robust to other misconduct databases, as outlined in our robustness section. For the restatement data, we obtain all restatements to earnings during our sample period and exclude those relating to i) retrospective revisions for comparability, ii) retrospective application of accounting principles, and iii) alternations in presentation due to mergers. MISCONDUCT is a dummy variable equal to 1 in the year in which either earnings restatements or the above legal actions are reported. MISCONDUCT identifies the fraudulent activity as of the time that the public becomes aware of the misconduct, not the time that the alleged misconduct occurs. We collect monetary settlement information for all misconduct cases. Among the 1,032 cases in our sample, the settlement amount ranges from $\$ 0$ to $\$ 68$ million, with a mean

\footnotetext{
${ }^{18}$ Bebchuk et al. (2009) develop a governance index (the E-index) based on 6 of the 24 provisions originally identified in Gompers et al. (2003). The E-index is compiled so that high values indicate stronger governance (i.e., fewer anti-takeover restrictions or lower managerial entrenchment). Our results are robust to using all of the provisions in Gompers et al.

${ }^{19} \mathrm{~A}$ natural question is whether the market reacts differently to the death of non-co-opted relative to co-opted AC directors. Of our 122 cases of director death, 67 involve non-co-opted directors and 55 involve co-opted directors. Univariate results show that the CAR averages $-1.6 \%(-1.2 \%)$ when a non-co-opted (co-opted) AC director dies. Regression results using specifications similar to model 1 of Table 4 indicate that the market responds negatively when any highly connected AC director dies. Specifically, we use two dummy variables to represent highly connected, non-co-opted directors and highly connected, co-opted directors. The coefficient on each dummy variable is significant and of magnitude $-3.1 \%$ and $-2.7 \%$, respectively. An $F$-test shows no difference between the two variables, although the small sample sizes of each subgroup likely results in low power. Although it would be interesting to also examine whether the observed negative market reaction is due to the loss of connections or from a change from non-co-opted to co-opted AC director, the successor is generally not known at the time of the announcement of the director's death.
} 
TABLE 4

Market Assessment of Director Connectedness: Evidence from Director Death

Table 4 examines the market reaction to audit committee member death cases. We identify 122 announcements of audit committee member deaths. See Appendix $D$ for variable definitions. Numbers and variables of interest are in bold. The $t$-statistics (in parentheses) are adjusted for heteroscedasticity using the Huber-White sandwich estimator and are corrected for clustering at the firm level. * and ** indicate significance at the $10 \%$ and $5 \%$ levels using 2-tailed tests, respectively.

\begin{tabular}{|c|c|c|c|}
\hline \multirow[b]{2}{*}{ Variable } & \multicolumn{3}{|c|}{ Dependent Variable: CAR } \\
\hline & 1 & 2 & 3 \\
\hline Constant & $\begin{array}{c}0.045 \\
(0.69)\end{array}$ & $\begin{array}{r}0.051 \\
(0.92)\end{array}$ & $\begin{array}{r}0.056 \\
(1.22)\end{array}$ \\
\hline HIGH_D_CONNECT & $\begin{array}{l}-0.030^{\star *} \\
(-2.51)\end{array}$ & $\begin{array}{l}-0.030^{\star *} \\
(-2.30)\end{array}$ & $\begin{array}{l}-0.028^{*} \\
(-2.25)\end{array}$ \\
\hline LOW_AC_CONNECT & - & $\begin{array}{r}-0.017 \\
(-1.10)\end{array}$ & - \\
\hline HIGH_D_CONNECT $\times$ LOW_AC_CONNECT & - & $\begin{array}{l}-0.011^{* *} \\
(-2.03)\end{array}$ & - \\
\hline POOR_GOVERN & - & - & $\begin{array}{l}-0.028 \\
(-1.23)\end{array}$ \\
\hline HIGH_D_CONNECT $\times$ POOR_GOVERN & - & - & $\begin{array}{l}-0.026^{\text {** }} \\
(-2.36)\end{array}$ \\
\hline HIGH_POST & $\begin{array}{c}0.003 \\
(0.59)\end{array}$ & $\begin{array}{c}0.005 \\
(0.71)\end{array}$ & $\begin{array}{r}0.003 \\
(0.63)\end{array}$ \\
\hline HIGH_EDU & $\begin{array}{l}0.005 \\
(0.81)\end{array}$ & $\begin{array}{l}0.002 \\
(0.22)\end{array}$ & $\begin{array}{l}0.003 \\
(0.52)\end{array}$ \\
\hline BOARD_SEATS & $\begin{array}{l}-0.002 \\
(-1.01)\end{array}$ & $\begin{array}{l}-0.002 \\
(-0.96)\end{array}$ & $\begin{array}{l}-0.001 \\
(-0.90)\end{array}$ \\
\hline D_AGE & $\begin{array}{l}-0.001 \\
(-0.21)\end{array}$ & $\begin{array}{l}-0.002 \\
(-0.21)\end{array}$ & $\begin{array}{l}-0.002 \\
(-0.25)\end{array}$ \\
\hline D_TENURE & $\begin{array}{r}0.007 \\
(0.31)\end{array}$ & $\begin{array}{r}0.005 \\
(0.42)\end{array}$ & $\begin{array}{l}0.006 \\
(0.45)\end{array}$ \\
\hline D_FEMALE & $\begin{array}{l}-0.027 \\
(-0.93)\end{array}$ & $\begin{array}{l}-0.035 \\
(-1.15)\end{array}$ & $\begin{array}{l}-0.029 \\
(-1.10)\end{array}$ \\
\hline ACCT_EXPERT & $\begin{array}{l}-0.009^{*} \\
(-1.72)\end{array}$ & $\begin{array}{l}-0.010^{*} \\
(-1.70)\end{array}$ & $\begin{array}{l}-0.011^{\star} \\
(-1.77)\end{array}$ \\
\hline AC_SIZE & $\begin{array}{l}-0.002 \\
(-0.21)\end{array}$ & $\begin{array}{l}-0.003 \\
(-0.33)\end{array}$ & $\begin{array}{l}-0.002 \\
(-0.25)\end{array}$ \\
\hline CEO_CONNECT & $\begin{array}{r}0.010 \\
(1.41)\end{array}$ & $\begin{array}{l}0.009^{*} \\
(1.69)\end{array}$ & $\begin{array}{l}0.011^{*} \\
(1.83)\end{array}$ \\
\hline CEO/D_OVERLAP & $\begin{array}{l}-0.011 \\
(-1.22)\end{array}$ & $\begin{array}{l}-0.010 \\
(-1.39)\end{array}$ & $\begin{array}{l}-0.010 \\
(-1.23)\end{array}$ \\
\hline In(FIRM_SIZE) & $\begin{array}{l}-0.002 \\
(-0.47)\end{array}$ & $\begin{array}{l}-0.003 \\
(-0.66)\end{array}$ & $\begin{array}{l}-0.003 \\
(-0.68)\end{array}$ \\
\hline VOLATILITY & $\begin{array}{l}-0.330^{\star \star} \\
(-2.03)\end{array}$ & $\begin{array}{l}-0.307^{\star \star} \\
(-2.15)\end{array}$ & $\begin{array}{l}-0.312^{\star \star} \\
(-2.22)\end{array}$ \\
\hline INST_OWN & $\begin{array}{l}-0.007 \\
(-0.38)\end{array}$ & $\begin{array}{l}-0.008 \\
(-0.35)\end{array}$ & $\begin{array}{l}-0.010 \\
(-0.70)\end{array}$ \\
\hline Year dummy & Yes & Yes & Yes \\
\hline $\begin{array}{l}\text { No. of obs. } \\
\text { Adj. } R^{2}\end{array}$ & $\begin{array}{r}122 \\
0.119\end{array}$ & $\begin{array}{r}122 \\
0.102\end{array}$ & $\begin{array}{r}122 \\
0.118\end{array}$ \\
\hline
\end{tabular}

(median) of $\$ 1.5$ million ( $\$ 0)$. Alternatively, we gauge the severity of the misconduct using the CAR around the misconduct announcement date. The mean (median) CAR during the $(-2,+2)$ window is $-6.38 \%(-4.93 \%)$. As discussed in Section II.B, all else equal, connected directors should have better labor opportunities in general, but how they fare following the detection of misconduct is an empirical question. We initially focus on independent, non-co-opted AC members and examine both director turnover and the procurement of additional board seats following misconduct. 
We begin by examining the effect of connectedness the likelihood of director turnover in the year following the discovery of fraudulent activity. ${ }^{20} \mathrm{We}$ control for a variety of director characteristics, including age, tenure, a dummy variable indicating whether the director is female, CEO/director overlap, number of highlevel positions, educational background, and number of external board seats. We also control for staggered boards, as a director on a staggered board is generally up for election only once every 3 years, as opposed to every year on a unitary board. Other firm-level control variables are similar to those examined in prior studies (Yermack (2004), Srinivasan (2005), and Fahlenbrach et al. (2017)). Lastly, we control for industry and year fixed effects. Panel A of Table 5 presents the results. Column 1 examines our sample of independent, non-co-opted AC director observations; our variables of interest are MISCONDUCT and HIGH_D_CONNECT. ${ }^{21}$ Consistent with our predictions and with prior studies, the coefficient on MISCONDUCT is positive and significant, indicating that misconduct is associated with a higher likelihood of AC member turnover. The negative and significant coefficient on HIGH_D_CONNECT suggests that, overall, highly connected directors are less likely to be dismissed from the board.

In column 2 of Table 5, we add an interaction term between HIGH_D_ CONNECT and MISCONDUCT.22 The coefficients of MISCONDUCT and HIGH_D_CONNECT remain significant. The coefficient on MISCONDUCT $\times$ HIGH_D_CONNECT is also negative and significant, indicating that when misconduct is detected, well-connected AC members face less turnover than less connected members. An $F$-test on the sum of MISCONDUCT and MISCONDUCT $\times$ HIGH_D_CONNECT is not significant, indicating that highly connected AC directors do not experience turnover following misconduct. The economic significance of our findings is strong, as indicated by the marginal effects; highly connected AC members have a $25.5 \%$ lower chance of turnover. Additionally, when misconduct occurs, these directors have an incremental $15.6 \%$ lower likelihood of turnover. $^{23}$

\footnotetext{
${ }^{20}$ To mitigate the possibility that well-connected directors choose to depart if they foresee trouble, we examine the frequency of departures in the 1-2 years before the detection of fraudulent activity. In untabulated results, we find that relative to other directors, highly connected directors are no more likely to depart before fraud.

${ }^{21}$ To facilitate interpretation, we use a dummy variable based on the sample distribution of director connectedness (HIGH_D_CONNECT) as one of our main variables of interest. To rule out the possibility that our results are driven by this particular form of measure, we replicate our analysis using a continuous measure of director connectedness. Untabulated results show that the continuous measure yields similar inferences as our main results.

${ }^{22} \mathrm{Ai}$ and Norton (2003) and Greene (2010) find that interaction terms in non-linear models can potentially result in biased estimates of the coefficient and the standard error. Consequently, for all the logit and Tobit models we use involving interaction terms, we recheck using an OLS regression and obtain similar results.

${ }^{23} \mathrm{An}$ alternative interpretation of our findings is that $\mathrm{AC}$ members from firms that have at least one well-connected AC member are less likely to be punished following fraud. In other words, our results could be driven by a firm-level effect instead of a director effect. To investigate such a possibility, we split our sample firms by the median level of AC connectedness and repeat our test for each subsample. We find that the effect of $\mathrm{AC}$ director connectedness on their turnover is more pronounced in the firms that have lower AC connectedness, suggesting that it is the individual director's connectedness that drives our findings.
} 


\section{Misconduct and Audit Committee Director Career Consequences}

Table 5 examines the effect of director connectedness on career consequences. Panel A reports the likelihood of director turnover in the year after the detection of misconduct, and Panel B reports the change in the number of board seats held by the director in the 3 years following the detection of fraudulent activity. Panels A and B examine non-co-opted audit committee directors, and Panel $\mathrm{C}$ includes all highly connected board members, defined as directors with top quartile connectedness measures. HIGH_D_CONNECT is an indicator equal to 1 if director connectedness is in the top quartile of the sample. AC is an indicator equal to 1 if the director is on the audit committee. For the propensity-score-matched sample, we match firms with detected misconduct to firms without based on the following variables: firm size, chief executive officer (CEO) power, CEO tenure, return on assets (ROA), volatility, institutional ownership, analyst following, audit committee size, audit committee independence, average audit committee director age, average director tenure, audit committee female ratio, FSCORE, and industry and year dummies. See Appendix D for all other variable definitions. Numbers and variables of interest are in bold. The $z(t)$-statistics (in parentheses) are adjusted for heteroscedasticity using the Huber-White sandwich estimator and are clustered at the firm level. Marginal effects at the mean are reported in square brackets. ${ }^{*},{ }^{\star *}$, and ${ }^{\star \star \star}$ indicate significance at the $10 \%, 5 \%$, and $1 \%$ levels using 2 -tailed tests, respectively.

Panel A. Logistic Regressions of Director Turnover

\begin{tabular}{|c|c|c|c|c|c|}
\hline \multirow[b]{3}{*}{ Variables } & \multicolumn{5}{|c|}{ Sample } \\
\hline & \multicolumn{2}{|c|}{ Full } & \multicolumn{2}{|c|}{ Matched } & \multirow{2}{*}{$\frac{\text { Misconduct Only }}{5}$} \\
\hline & 1 & 2 & 3 & 4 & \\
\hline Constant & $\begin{array}{l}-3.099^{\star \star \star} \\
(-4.56)\end{array}$ & $\begin{array}{l}-2.525^{\star \star \star} \\
(-4.19)\end{array}$ & $\begin{array}{l}-21.903^{\star \star \star} \\
(-22.01)\end{array}$ & $\begin{array}{l}-20.129^{\star \star \star} \\
(-21.82)\end{array}$ & $\begin{array}{l}-21.346^{\star \star \star} \\
(-21.99)\end{array}$ \\
\hline $\operatorname{MISCONDUCT}\left(\beta_{3}\right)$ & $\begin{array}{l}0.195^{*}[0.233] \\
(1.82)\end{array}$ & $\begin{array}{l}0.189^{*}[0.209] \\
\quad(1.92)\end{array}$ & $\begin{array}{l}0.299^{\star \star}[0.346] \\
(2.39)\end{array}$ & $\begin{array}{l}0.288^{\star \star}[0.335] \\
(2.50)\end{array}$ & - \\
\hline HIGH_D_CONNECT $\left(\boldsymbol{\beta}_{1}\right)$ & $\begin{array}{c}-0.466^{\star \star \star}[-0.283] \\
(-16.39)\end{array}$ & $\begin{array}{l}-0.429^{\star \star \star}[-0.255] \\
(-17.79)\end{array}$ & $\begin{array}{c}-0.457^{\star \star}[-0.259] \\
(-2.29)\end{array}$ & $\begin{array}{l}-0.433^{\star *}[-0.241] \\
(-2.11)\end{array}$ & $\begin{array}{c}-0.506^{\star \star \star}[-0.280] \\
(-2.90)\end{array}$ \\
\hline $\begin{array}{l}\text { MISCONDUCT } \times \\
\text { HIGH_D_CONNECT }\left(\beta_{2}\right)\end{array}$ & - & $\begin{array}{l}-0.129^{\star \star}[-0.156] \\
\quad(-2.33)\end{array}$ & - & $\begin{array}{l}-0.220^{\star \star}[-0.141] \\
(-2.49)\end{array}$ & - \\
\hline HIGH_POST & $\begin{array}{l}-0.029^{* \star \star} \\
(-5.01)\end{array}$ & $\begin{array}{l}-0.031^{\star \star \star} \\
(-5.26)\end{array}$ & $\begin{array}{l}-0.030 \\
(-1.10)\end{array}$ & $\begin{array}{l}-0.035 \\
(-1.02)\end{array}$ & $\begin{array}{l}-0.031 \\
(-0.77)\end{array}$ \\
\hline HIGH_EDU & $\begin{array}{l}-0.056 \\
(-0.87)\end{array}$ & $\begin{array}{l}-0.049 \\
(-0.90)\end{array}$ & $\begin{array}{l}-0.150 \\
(-0.59)\end{array}$ & $\begin{array}{l}-0.152 \\
(-0.55)\end{array}$ & $\begin{array}{l}-0.123 \\
(-0.45)\end{array}$ \\
\hline BOARD_SEATS & $\begin{array}{l}-1.426^{\star \star \star} \\
(-3.80)\end{array}$ & $\begin{array}{l}-1.413^{\star \star \star} \\
(-3.76)\end{array}$ & $\begin{array}{l}-1.352^{\star \star \star} \\
(-6.27)\end{array}$ & $\begin{array}{l}-1.269^{\star \star \star} \\
(-6.33)\end{array}$ & $\begin{array}{l}-1.855^{\star \star \star} \\
(-6.87)\end{array}$ \\
\hline D_AGE & $\begin{array}{l}0.010^{* \star \star} \\
(13.29)\end{array}$ & $\begin{array}{l}0.011^{\star \star \star} \\
(14.56)\end{array}$ & $\begin{array}{l}0.020^{\star \star \star \star} \\
(2.79)\end{array}$ & $\begin{array}{l}0.017^{\star \star \star} \\
(2.75)\end{array}$ & $\begin{array}{r}0.006 \\
(1.12)\end{array}$ \\
\hline D_FEMALE & $\begin{array}{l}-0.020 \\
(-0.56)\end{array}$ & $\begin{array}{l}-0.024 \\
(-0.52)\end{array}$ & $\begin{array}{l}0.572^{\star \star \star} \\
(2.70)\end{array}$ & $\begin{array}{l}0.582^{\star \star \star} \\
(2.75)\end{array}$ & $\begin{array}{l}0.680^{\star \star \star} \\
(2.85)\end{array}$ \\
\hline D_TENURE & $\begin{array}{l}0.105^{\star \star \star} \\
(6.49)\end{array}$ & $\begin{array}{l}0.099^{\star \star \star} \\
(6.45)\end{array}$ & $\begin{array}{c}0.036 \\
(0.50)\end{array}$ & $\begin{array}{c}0.040 \\
(0.67)\end{array}$ & $\begin{array}{l}0.111 \\
(0.99)\end{array}$ \\
\hline AC_SIZE & $\begin{array}{l}-0.005 \\
(-1.30)\end{array}$ & $\begin{array}{l}-0.005 \\
(-1.35)\end{array}$ & $\begin{array}{l}-0.004 \\
(-1.46)\end{array}$ & $\begin{array}{l}-0.004 \\
(-1.52)\end{array}$ & $\begin{array}{l}-0.007 \\
(-1.41)\end{array}$ \\
\hline AC_INDEPENDENCE & $\begin{array}{c}0.158 \\
(1.43)\end{array}$ & $\begin{array}{c}0.152 \\
(1.50)\end{array}$ & $\begin{array}{c}0.333 \\
(1.50)\end{array}$ & $\begin{array}{c}0.350 \\
(1.49)\end{array}$ & $\begin{array}{c}0.543 \\
(1.30)\end{array}$ \\
\hline CEO_CONNECT & $\begin{array}{r}0.031 \\
(1.37)\end{array}$ & $\begin{array}{c}0.033 \\
(1.26)\end{array}$ & $\begin{array}{r}0.017 \\
(0.89)\end{array}$ & $\begin{array}{c}0.016 \\
(0.78)\end{array}$ & $\begin{array}{c}0.062 \\
(0.72)\end{array}$ \\
\hline CEO/D_OVERLAP & $\begin{array}{l}-1.762^{\star \star \star} \\
(-6.67)\end{array}$ & $\begin{array}{l}-1.833^{\star \star \star} \\
(-7.01)\end{array}$ & $\begin{array}{l}-0.932 \\
(-0.81)\end{array}$ & $\begin{array}{l}-0.955 \\
(-0.90)\end{array}$ & $\begin{array}{l}-1.110^{\star \star \star} \\
(-2.60)\end{array}$ \\
\hline In(FIRM_SIZE) & $\begin{array}{l}0.090 * \star \star \\
(12.96)\end{array}$ & $\begin{array}{l}0.091^{\star \star \star} \\
(13.02)\end{array}$ & $\begin{array}{l}0.230^{\star \star \star} \\
(4.75)\end{array}$ & $\begin{array}{l}0.223^{\star \star \star} \\
(4.70)\end{array}$ & $\begin{array}{l}0.203^{\star \star \star} \\
(3.45)\end{array}$ \\
\hline STAGGERED_BOARD & $\begin{array}{l}-0.072^{*} \\
(-1.80)\end{array}$ & $\begin{array}{l}-0.077^{\star} \\
(-1.89)\end{array}$ & $\begin{array}{l}-0.232^{*} \\
(-1.88)\end{array}$ & $\begin{array}{l}-0.240^{\star} \\
(-1.85)\end{array}$ & $\begin{array}{l}-0.259^{\star} \\
(-1.72)\end{array}$ \\
\hline ANALYSTS & $\begin{array}{l}-0.001 \\
(-0.85)\end{array}$ & $\begin{array}{l}-0.001 \\
(-0.79)\end{array}$ & $\begin{array}{l}-0.029^{\star \star \star} \\
(-2.59)\end{array}$ & $\begin{array}{l}-0.025^{\star \star} \\
(-2.50)\end{array}$ & $\begin{array}{l}-0.017 \\
(-1.33)\end{array}$ \\
\hline VOLATILITY & $\begin{array}{l}0.509^{\star \star \star} \\
(2.72)\end{array}$ & $\begin{array}{l}0.522^{\star \star \star} \\
(2.99)\end{array}$ & $\begin{array}{l}1.105 \\
(1.20)\end{array}$ & $\begin{array}{l}1.129 \\
(1.22)\end{array}$ & $\begin{array}{r}0.081 \\
(1.12)\end{array}$ \\
\hline INST_OWN & $\begin{array}{l}-0.065 \\
(-1.50)\end{array}$ & $\begin{array}{l}-0.059 \\
(-1.34)\end{array}$ & $\begin{array}{l}-0.330 \\
(-0.99)\end{array}$ & $\begin{array}{l}-0.322 \\
(-0.90)\end{array}$ & $\begin{array}{l}-0.146 \\
(-0.49)\end{array}$ \\
\hline PRIOR_RET & $\begin{array}{l}-0.052^{\star \star \star} \\
(-4.60)\end{array}$ & $\begin{array}{l}-0.055^{\star \star \star} \\
(-4.66)\end{array}$ & $\begin{array}{l}-0.092 \\
(-1.32)\end{array}$ & $\begin{array}{l}-0.089 \\
(-1.23)\end{array}$ & $\begin{array}{l}-0.166 \\
(-1.27)\end{array}$ \\
\hline $\begin{array}{l}F \text {-test } p \text {-value: } \beta_{1}+\beta_{2}=0 \\
\text { F-test } p \text {-value: } \beta_{2}+\beta_{3}=0\end{array}$ & - & $\begin{array}{l}0.000 \\
0.356\end{array}$ & - & $\begin{array}{l}0.000 \\
0.459\end{array}$ & - \\
\hline Industry and year dummies & Yes & Yes & Yes & Yes & Yes \\
\hline No. of obs. & 31,344 & 31,344 & 3,712 & 3,712 & 2,065 \\
\hline Pseudo- $R^{2}$ & 0.198 & 0.199 & 0.266 & 0.268 & 0.322 \\
\hline
\end{tabular}


TABLE 5 (continued)

Misconduct and Audit Committee Director Career Consequences

\begin{tabular}{|c|c|c|c|c|c|c|}
\hline \multirow[b]{3}{*}{ Variables } & \multicolumn{6}{|c|}{ Sample } \\
\hline & \multicolumn{2}{|r|}{ Full } & \multicolumn{3}{|c|}{ Matched } & \multirow{2}{*}{$\begin{array}{c}\text { Misconduct Only } \\
5\end{array}$} \\
\hline & 1 & 2 & 3 & & 4 & \\
\hline Constant & $\begin{array}{l}1.156^{\star \star \star *} \\
(5.82)\end{array}$ & $\begin{array}{l}1.028^{\star \star \star \star} \\
(5.66)\end{array}$ & $\begin{array}{l}2.366^{\star \star \star} \\
(3.39)\end{array}$ & & $\begin{array}{l}2.552^{\star \star \star} \\
(3.43)\end{array}$ & $\begin{array}{c}0.526 \\
(1.02)\end{array}$ \\
\hline $\operatorname{MISCONDUCT}\left(\beta_{3}\right)$ & $\begin{array}{l}-0.139^{\star \star} \\
(-2.36)\end{array}$ & $\begin{array}{l}-0.133^{\star \star} \\
(-2.29)\end{array}$ & $\begin{array}{l}-0.095^{\star \star} \\
(-2.55)\end{array}$ & & $\begin{array}{l}-0.090^{\star \star} \\
(-2.50)\end{array}$ & - \\
\hline HIGH_D_CONNECT $\left(\boldsymbol{\beta}_{1}\right)$ & $\begin{array}{l}0.556^{* \star *} \\
(3.30)\end{array}$ & $\begin{array}{l}0.536^{\star \star \star} \\
(3.19)\end{array}$ & $\begin{array}{l}0.532^{\star \star \star} \\
(2.93)\end{array}$ & & $\begin{array}{l}0.522^{\star \star \star} \\
(2.90)\end{array}$ & $\begin{array}{l}0.212^{\star \star} \\
(2.51)\end{array}$ \\
\hline $\begin{array}{l}\text { MISCONDUCT } x \\
\text { HIGH_D_CONNECT }\left(\beta_{2}\right)\end{array}$ & - & $\begin{array}{l}0.456^{* \star *} \\
(3.33)\end{array}$ & - & & $\begin{array}{l}0.409^{\star *} \\
(2.36)\end{array}$ & - \\
\hline HIGH_POST & $\begin{array}{l}0.036^{\star \star \star} \\
(2.73)\end{array}$ & $\begin{array}{l}0.039^{\star \star \star} \\
(2.87)\end{array}$ & $\begin{array}{c}0.028 \\
(0.88)\end{array}$ & & $\begin{array}{l}0.030 \\
(0.90)\end{array}$ & $\begin{array}{r}0.041 \\
(1.23)\end{array}$ \\
\hline HIGH_EDU & $\begin{array}{l}0.490^{\star \star \star \star} \\
(6.30)\end{array}$ & $\begin{array}{l}0.477^{\star \star \star} \\
(6.12)\end{array}$ & $\begin{array}{l}0.236 \\
(0.89)\end{array}$ & & $\begin{array}{r}0.230 \\
(0.86)\end{array}$ & $\begin{array}{l}0.432 \\
(1.10)\end{array}$ \\
\hline BOARD_SEATS & $\begin{array}{l}0.460^{\star \star \star} \\
(45.55)\end{array}$ & $\begin{array}{l}0.4677^{\star \star \star} \\
(45.90)\end{array}$ & $\begin{array}{l}0.626^{\star \star \star} \\
(17.92)\end{array}$ & & $\begin{array}{l}0.611^{\star \star \star} \\
(17.79)\end{array}$ & $\begin{array}{l}0.655^{\star \star \star} \\
(17.00)\end{array}$ \\
\hline D_AGE & $\begin{array}{l}-0.011^{\star \star \star} \\
(-18.28)\end{array}$ & $\begin{array}{l}-0.010^{\star \star \star} \\
(-18.90)\end{array}$ & $\begin{array}{l}-0.013^{\star \star \star} \\
(-3.07)\end{array}$ & & $\begin{array}{l}-0.014^{\star \star \star} \\
(-3.19)\end{array}$ & $\begin{array}{l}-0.007 \\
(-1.45)\end{array}$ \\
\hline D_FEMALE & $\begin{array}{l}-0.033 \\
(-0.67)\end{array}$ & $\begin{array}{l}-0.037 \\
(-0.78)\end{array}$ & $\begin{array}{l}-0.547^{\star \star} \\
(-2.55)\end{array}$ & & $\begin{array}{l}-0.535^{\star \star} \\
(-2.52)\end{array}$ & $\begin{array}{l}-0.088 \\
(-0.50)\end{array}$ \\
\hline T_TENURE & $\begin{array}{l}-0.155^{\star \star} \\
(-2.50)\end{array}$ & $\begin{array}{l}-0.150^{\star \star} \\
(-2.52)\end{array}$ & $\begin{array}{l}-0.028 \\
(-0.33)\end{array}$ & & $\begin{array}{l}-0.030 \\
(-0.35)\end{array}$ & $\begin{array}{l}-0.052 \\
(-0.50)\end{array}$ \\
\hline AC_SIZE & $\begin{array}{l}0.001^{\star} \\
(1.85)\end{array}$ & $\begin{array}{l}0.001^{\star} \\
(1.88)\end{array}$ & $\begin{array}{c}0.001 \\
(0.55)\end{array}$ & & $\begin{array}{r}0.001 \\
(0.47)\end{array}$ & $\begin{array}{r}0.001 \\
(0.73)\end{array}$ \\
\hline AC_INDEPENDENCE & $\begin{array}{l}-0.437 \\
(-1.42)\end{array}$ & $\begin{array}{l}-0.455 \\
(-1.55)\end{array}$ & $\begin{array}{l}-0.437^{\star} \\
(-1.73)\end{array}$ & & $\begin{array}{l}-0.446^{*} \\
(-1.71)\end{array}$ & $\begin{array}{l}-0.511^{\star} \\
(-1.69)\end{array}$ \\
\hline CEO_CONNECT & $\begin{array}{l}0.045 \\
(0.90)\end{array}$ & $\begin{array}{c}0.039 \\
(0.78)\end{array}$ & $\begin{array}{c}0.036 \\
(0.78)\end{array}$ & & $\begin{array}{c}0.032 \\
(0.66)\end{array}$ & $\begin{array}{l}0.023 \\
(0.52)\end{array}$ \\
\hline CEO/D_OVERLAP & $\begin{array}{l}0.355^{*} \\
(1.82)\end{array}$ & $\begin{array}{l}0.350^{*} \\
(1.80)\end{array}$ & $\begin{array}{r}0.426 \\
(1.45)\end{array}$ & & $\begin{array}{r}0.418 \\
(1.36)\end{array}$ & $\begin{array}{l}0.322 \\
(1.41)\end{array}$ \\
\hline In(FIRM_SIZE) & $\begin{array}{l}0.096^{\star \star \star} \\
(3.37)\end{array}$ & $\begin{array}{l}0.102^{\star \star \star} \\
(3.60)\end{array}$ & $\begin{array}{c}0.035 \\
(1.63)\end{array}$ & & $\begin{array}{c}0.033 \\
(1.60)\end{array}$ & $\begin{array}{l}0.129^{\star \star} \\
(2.11)\end{array}$ \\
\hline ANALYSTS & $\begin{array}{l}0.017^{\star} \\
(1.88)\end{array}$ & $\begin{array}{l}0.018^{\star} \\
(1.90)\end{array}$ & $\begin{array}{r}0.007 \\
(0.87)\end{array}$ & & $\begin{array}{l}0.006 \\
(0.61)\end{array}$ & $\begin{array}{l}0.005 \\
(0.47)\end{array}$ \\
\hline VOLATILITY & $\begin{array}{l}-3.409^{\star \star \star} \\
(-3.26)\end{array}$ & $\begin{array}{l}-3.355^{\star \star \star} \\
(-3.00)\end{array}$ & $\begin{array}{l}-3.822^{\star \star \star} \\
(-4.79)\end{array}$ & & $\begin{array}{l}-3.859^{\star \star \star} \\
(-4.90)\end{array}$ & $\begin{array}{l}-0.700 \\
(-0.86)\end{array}$ \\
\hline INST_OWN & $\begin{array}{l}-0.422^{\star \star \star} \\
(-2.76)\end{array}$ & $\begin{array}{l}-0.433^{\star \star \star} \\
(-2.98)\end{array}$ & $\begin{array}{l}-0.439^{\star} \\
(-1.85)\end{array}$ & & $\begin{array}{l}-0.436^{*} \\
(-1.77)\end{array}$ & $\begin{array}{l}-0.177 \\
(-0.71)\end{array}$ \\
\hline $\begin{array}{l}F \text {-test } p \text {-value: } \beta_{1}+\beta_{2}=0 \\
F \text {-test } p \text {-value: } \beta_{2}+\beta_{3}=0\end{array}$ & $\overline{-}$ & $\begin{array}{l}0.000 \\
0.002\end{array}$ & $\begin{array}{l}- \\
-\end{array}$ & & $\begin{array}{l}0.000 \\
0.010\end{array}$ & $\begin{array}{l}- \\
-\end{array}$ \\
\hline Industry and year dummies & Yes & Yes & Yes & & Yes & Yes \\
\hline $\begin{array}{l}\text { No. of obs. } \\
\text { Pseudo- } R^{2}\end{array}$ & $\begin{array}{r}31,344 \\
0.035\end{array}$ & $\begin{array}{r}31,344 \\
0.035\end{array}$ & $\begin{array}{l}3,712 \\
0.063\end{array}$ & & $\begin{array}{l}3,712 \\
0.063\end{array}$ & $\begin{array}{l}2,065 \\
0.083\end{array}$ \\
\hline \multicolumn{7}{|c|}{ Panel C. Director Turnover and Future Board Seats: Highly Connected AC and Highly Connected Non-AC Directors } \\
\hline & \multicolumn{6}{|c|}{ Dependent Variable } \\
\hline & \multicolumn{3}{|c|}{ Turnover } & \multicolumn{3}{|c|}{ Future Board Seats } \\
\hline & \multicolumn{6}{|c|}{ Sample } \\
\hline & Full & Matched & $\begin{array}{l}\text { Misconduct } \\
\text { Only }\end{array}$ & Full & Matched & $\begin{array}{l}\text { Misconduct } \\
\text { Only }\end{array}$ \\
\hline Variables & 1 & 2 & 3 & 4 & 5 & 6 \\
\hline $\mathrm{AC}\left(\beta_{1}\right)$ & $\begin{array}{l}1.339 \\
(1.60)\end{array}$ & $\begin{array}{l}1.679^{\star} \\
(1.90)\end{array}$ & $\begin{array}{l}1.902^{\star *} \\
(2.50)\end{array}$ & $\begin{array}{l}0.543^{\star \star} \\
(2.33)\end{array}$ & $\begin{array}{l}0.578^{* \star} \\
(2.52)\end{array}$ & $\begin{array}{l}-0.018 \\
(-0.59)\end{array}$ \\
\hline MISCONDUCT $\left(\beta_{2}\right)$ & $\begin{array}{l}0.161^{*} \\
(1.70)\end{array}$ & $\begin{array}{c}0.170^{*} \\
(1.72)\end{array}$ & - & $\begin{array}{l}-0.409^{\star \star} \\
(-2.28)\end{array}$ & $\begin{array}{l}-0.322^{\star \star} \\
(-2.50)\end{array}$ & - \\
\hline AC $\times$ MISCONDUCT $\left(\beta_{3}\right)$ & $\begin{array}{l}2.005^{\star \star \star} \\
(2.89)\end{array}$ & $\begin{array}{l}2.262^{\star \star \star} \\
(2.90)\end{array}$ & - & $\begin{array}{l}-0.887^{\star \star} \\
(-2.35)\end{array}$ & $\begin{array}{l}-0.804^{* \star} \\
(-2.22)\end{array}$ & - \\
\hline $\begin{array}{l}F \text {-test } p \text {-value: } \beta_{1}+\beta_{2}=0 \\
F \text {-test } p \text {-value: } \beta_{2}+\beta_{3}=0\end{array}$ & $\begin{array}{l}18.93^{\star \star \star} \\
19.14^{\star \star \star}\end{array}$ & $\begin{array}{l}22.36^{\star \star \star} \\
19.16^{\star \star \star}\end{array}$ & 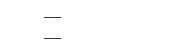 & $\begin{array}{l}1.20 \\
19.23^{\star \star \star}\end{array}$ & $\begin{array}{l}0.56 \\
17.16^{\star \star \star}\end{array}$ & 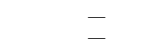 \\
\hline $\begin{array}{l}\text { Controls } \\
\text { Industry and year dummies }\end{array}$ & $\begin{array}{l}\text { Yes } \\
\text { Yes }\end{array}$ & $\begin{array}{l}\text { Yes } \\
\text { Yes }\end{array}$ & $\begin{array}{l}\text { Yes } \\
\text { Yes }\end{array}$ & $\begin{array}{l}\text { Yes } \\
\text { Yes }\end{array}$ & $\begin{array}{l}\text { Yes } \\
\text { Yes }\end{array}$ & $\begin{array}{l}\text { Yes } \\
\text { Yes }\end{array}$ \\
\hline No. of obs. & 29,216 & 2,322 & 1,192 & 29,216 & 2,322 & 1,192 \\
\hline Pseudo- $R^{2}$ & 0.230 & 0.251 & 0.390 & 0.041 & 0.059 & 0.095 \\
\hline
\end{tabular}


To alleviate the concern that $\mathrm{AC}$ members self-select to serve on the boards of specific types of firms, we use a propensity-score-matching approach on the 1,032 firms with detected misconduct (refer to Appendix E for a description of the matching procedure). Using one-to-one matching with a caliper of $0.1 \%$ without replacement, we are able to match 928 firms with misconduct to firms without. ${ }^{24}$ Columns 3 and 4 of Table 5 examine director turnover in the matched sample. Similar to the findings in columns 1 and 2, the matched sample results suggest that i) AC members with a high level of connectedness are $24.1 \%-25.9 \%$ less prone to turnover and ii) in the case of misconduct, AC members with greater connectedness face $14.1 \%$ less chance of turnover than less connected AC directors. Column 5 limits the sample to firms that experience fraudulent activity; the results are consistent with those in columns 1-4. On average, an AC member with high connectedness is $28 \%$ less likely to experience turnover after misconduct. ${ }^{25}$

In Panel B of Table 5, we further examine the career prospects of directors by studying the likelihood of AC members obtaining board seats at other firms following the detection of misconduct. Our dependent variable is the change in the total number of board seats held by the director in the 3 years following the detection of fraudulent activity. We use a Tobit model to account for the censored nature of the dependent variable. ${ }^{26}$ As in Panel A, we report results on the full, matched, and misconduct-only samples in columns 1-5, respectively. Examination of the full sample indicates that on average, AC members lose board seats following misconduct, which is consistent with Srinivasan (2005) and Fich and Shivdasani (2007). The positive and significant coefficient on HIGH_D_CONNECT suggests that better connected AC members obtain additional future board seats, all else equal. Moreover, after the discovery of misconduct, these directors obtain relatively more board seats than AC members with low connectedness, as shown by the positive and significant coefficient on the MISCONDUCT $\times$ HIGH_D_CONNECT interaction. An $F$-test on the sum of MISCONDUCT and MISCONDUCT $\times$ HIGH_D_CONNECT is positive and significant, indicating that unlike less connected directors, highly connected AC directors tend to gain, rather than lose, board seats after the detection of misconduct. Results in columns 3-5 provide similar evidence. These findings suggest that better connected AC directors have more external opportunities and are less likely to be punished by the labor market, even after a monitoring failure is detected. ${ }^{27}$

Although the results in Panels A and B of Table 5 appear inconsistent with ex post settling up, the sample is restricted to $\mathrm{AC}$ members to isolate the impact

\footnotetext{
${ }^{24}$ In Appendix E, we show that our matching process yields a matched sample with similar characteristics. Using the Abadie-Imbens (2006) correction yields a similar matched sample.

${ }^{25}$ In untabulated analysis, we find that higher AC connectedness is associated with lower chance of fraud/misconduct. The effect is significantly more pronounced in smaller firms and firms with a non-busy AC, where self-selection is less likely to be a concern. These findings are consistent with the financial reporting quality results in Table 2 .

${ }^{26}$ To ensure that our results are not driven by outliers, we use a dummy variable to identify an increase in the number of board seats over the 3 years following the detection of fraud. Results are similar when using this measure.

${ }^{27}$ The results are reminiscent of Harford and Schonlau (2013), who find that directors who have been through merger and acquisition (M\&A) transactions are valued for that experience. Likewise, connected directors who have been through financial misconduct may be valued for their experience.
} 
of connectedness. Srinivasan (2005) notes that AC directors are more likely than non-AC directors to experience turnover following the detection of financial misconduct. If AC members are held more accountable for financial misconduct than non-AC members, our decision to limit the sample to AC members potentially affects the inferences drawn. Consequently, we also compare the career consequences of well-connected AC members to those of well-connected non-AC members, which allows us to hold connectedness constant and examine the impact of misconduct and $\mathrm{AC}$ membership on career consequences. We use a dummy variable (AC) to indicate whether the (highly connected) director is on the AC. We present the results in Panel C.

The results for the full sample (column 1 of Table 5) show that wellconnected AC directors are not more likely to experience turnover than wellconnected non-AC directors. Directors at firms with misconduct are more likely to depart, regardless of AC membership. The interaction term $(\mathrm{AC} \times$ MISCONDUCT) is positive and significant, indicating that $\mathrm{AC}$ members are more likely to experience turnover following misconduct than non-AC directors. This result is consistent with previous studies, such as Srinivasan (2005), that find that AC members are held particularly accountable when a firm experiences financial misconduct. Column 2 presents similar results for the propensity-score-matched sample. In column 3, we include only firms with misconduct and find that AC members are more likely to face turnover than non-AC directors.

Columns 4-6 of Table 5 reexamine the results on future board seats. Column 4 shows that well-connected $\mathrm{AC}$ members obtain more future board seats than well-connected non-AC directors. Directors are penalized if their firm experiences financial misconduct, however. The interaction $(\mathrm{AC} \times$ MISCONDUCT) shows that $\mathrm{AC}$ members are penalized more severely than non-AC members following misconduct. We observe similar results in the matched sample, shown in column 5. These results are again consistent with previous studies that find evidence of ex post settling up in the director labor market. Lastly, for the sample of misconduct firms, highly connected AC and non-AC members seem to be penalized by the labor market equally. Overall, results from Table 5 suggest that connectedness improves the career prospects of AC directors, even in the event of misconduct. Nonetheless, highly connected AC directors face career consequences and thus are held accountable in the event of financial misconduct. This combined analysis provides a more complete picture on the effect of director connectedness on their career consequences.

Finally, we explore whether the effect of director connectedness varies with the severity of misconduct. The reputation hypothesis suggests that diligent directors establish better reputations in the labor market (Fama (1980), Fama and Jensen (1983)). Financial misconduct may signal a monitoring failure by the AC; this failure may subsequently hinder the members' career prospects. We proxy for the severity of financial misconduct using 2 measures: i) the CARs during the 5 -day window $(-2,+2)$ surrounding the misconduct revelation date and ii) the case settlement amount (Fich and Shivdasani (2007)). ${ }^{28}$ Table 6 shows that the

\footnotetext{
${ }^{28}$ In the misconduct sample, the average CAR is $-6.38 \%$ and the lower quartile is $-12.41 \%$. For settlement amount, the average is roughly $\$ 1.5$ million and the top quartile is $\$ 2.65$ million. We
} 
TABLE 6

\section{Severity of Financial Misconduct and Director Connectedness}

Table 6 presents results of the effect of director connectedness on director turnover and future board seats after financial misconduct is detected at the firm level. In columns 1 and 3 , we use a dummy variable based on the top quartile monetary settlement amount for the severity of misconduct. In columns 2 and 4 , we use a dummy variable indicating top quartile announcement cumulative abnormal return (CAR) to gauge the severity of the misconduct. In columns 1 and 2, we use logit regression, and in columns 3 and 4, we use Tobit regression. See Appendix D for variable definitions. Numbers and variables of interest are in bold. The $z(t)$-statistics (in parentheses) are adjusted for heteroscedasticity using the HuberWhite sandwich estimator and are corrected for clustering at the firm level. Marginal effects at the mean are reported in square brackets. *, ${ }^{* *}$, and ${ }^{* * *}$ indicate significance at the $10 \%, 5 \%$, and $1 \%$ levels using 2 -tailed tests, respectively.

\begin{tabular}{|c|c|c|c|c|}
\hline \multirow[b]{4}{*}{ Variables } & \multicolumn{4}{|c|}{ Misconduct-Only Sample } \\
\hline & \multicolumn{4}{|c|}{ Dependent Variable } \\
\hline & \multicolumn{2}{|c|}{ Director Turnover } & \multicolumn{2}{|c|}{ Future Boards } \\
\hline & 1 & 2 & 3 & 4 \\
\hline Constant & $\begin{array}{l}-17.821^{\star \star \star} \\
(-19.09)\end{array}$ & $\begin{array}{l}-19.222^{\star \star \star} \\
(-18.67)\end{array}$ & $\begin{array}{l}0.450 \\
(0.70)\end{array}$ & $\begin{array}{r}0.447 \\
(0.82)\end{array}$ \\
\hline S_MISCONDUCT $\left(\boldsymbol{\beta}_{3}\right)$ & $\begin{array}{l}0.266^{\star \star}[0.192] \\
\quad(2.26)\end{array}$ & $\begin{array}{l}0.206^{\star \star}[0.207] \\
\quad(2.33)\end{array}$ & $\begin{array}{l}-0.250^{\star \star} \\
(-2.20)\end{array}$ & $\begin{array}{l}-0.242^{\star \star} \\
(-2.42)\end{array}$ \\
\hline HIGH_D_CONNECT $\left(\boldsymbol{\beta}_{1}\right)$ & $\begin{array}{l}-0.222^{\star \star}[-0.198] \\
\quad(-2.28)\end{array}$ & $\begin{array}{l}-0.260^{\star \star}[-0.277] \\
(-2.33)\end{array}$ & $\begin{array}{l}0.229^{\star \star} \\
(2.40)\end{array}$ & $\begin{array}{l}0.270^{\star \star \star} \\
(2.62)\end{array}$ \\
\hline $\begin{array}{l}\text { HIGH_D_CONNECT x } \\
\text { S_MISCONDUCT }\left(\boldsymbol{\beta}_{2}\right)\end{array}$ & $\begin{array}{l}-0.566^{\star \star}[-0.226] \\
\quad(-2.23)\end{array}$ & $\begin{array}{l}-0.457^{\star \star}[-0.250] \\
(-2.37)\end{array}$ & $\begin{array}{l}0.266^{\star \star} \\
(2.19)\end{array}$ & $\begin{array}{l}0.231^{\star \star} \\
(2.36)\end{array}$ \\
\hline HIGH_POST & $\begin{array}{l}-0.033 \\
(-0.85)\end{array}$ & $\begin{array}{l}-0.012 \\
(-0.29)\end{array}$ & $\begin{array}{r}0.041 \\
(1.21)\end{array}$ & $\begin{array}{c}0.044 \\
(1.33)\end{array}$ \\
\hline HIGH_EDU & $\begin{array}{l}-0.077 \\
(-0.29)\end{array}$ & $\begin{array}{l}-0.020 \\
(-0.12)\end{array}$ & $\begin{array}{c}0.432 \\
(1.21)\end{array}$ & $\begin{array}{r}0.421 \\
(1.06)\end{array}$ \\
\hline BOARD_SEATS & $\begin{array}{l}-1.838^{* \star \star} \\
(-6.55)\end{array}$ & $\begin{array}{l}-1.856^{\star \star \star} \\
(-6.80)\end{array}$ & $\begin{array}{l}0.659^{\star \star \star} \\
(15.78)\end{array}$ & $\begin{array}{l}0.657^{* * *} \\
(16.01)\end{array}$ \\
\hline D_AGE & $\begin{array}{l}0.005 \\
(0.88)\end{array}$ & $\begin{array}{l}0.005 \\
(0.82)\end{array}$ & $\begin{array}{l}-0.005 \\
(-1.08)\end{array}$ & $\begin{array}{l}-0.005 \\
(-1.11)\end{array}$ \\
\hline D_FEMALE & $\begin{array}{l}0.666^{* * *} \\
(2.66)\end{array}$ & $\begin{array}{l}0.671^{\star \star \star} \\
(2.89)\end{array}$ & $\begin{array}{l}-0.071 \\
(-0.45)\end{array}$ & $\begin{array}{l}-0.071 \\
(-0.36)\end{array}$ \\
\hline D_TENURE & $\begin{array}{l}0.111 \\
(0.92)\end{array}$ & $\begin{array}{l}0.115 \\
(1.02)\end{array}$ & $\begin{array}{l}-0.050 \\
(-0.66)\end{array}$ & $\begin{array}{l}-0.050 \\
(-0.62)\end{array}$ \\
\hline AC_SIZE & $\begin{array}{l}-0.006^{* *} \\
(-2.18)\end{array}$ & $\begin{array}{l}-0.004^{\star \star} \\
(-2.09)\end{array}$ & $\begin{array}{r}0.001 \\
(0.73)\end{array}$ & $\begin{array}{l}0.001 \\
(0.88)\end{array}$ \\
\hline AC_INDEPENDENCE & $\begin{array}{l}0.855 \\
(1.30)\end{array}$ & $\begin{array}{c}0.981 \\
(1.50)\end{array}$ & $\begin{array}{l}-0.471 \\
(-1.40)\end{array}$ & $\begin{array}{l}-0.466 \\
(-1.32)\end{array}$ \\
\hline CEO_CONNECT & $\begin{array}{l}0.055 \\
(0.82)\end{array}$ & $\begin{array}{l}0.059 \\
(0.98)\end{array}$ & $\begin{array}{l}0.022 \\
(0.69)\end{array}$ & $\begin{array}{l}0.020 \\
(0.71)\end{array}$ \\
\hline CEO/D_OVERLAP & $\begin{array}{l}-1.123^{\star *} \\
(-2.51)\end{array}$ & $\begin{array}{l}-1.222^{\star \star} \\
(-2.29)\end{array}$ & $\begin{array}{l}0.312 \\
(1.20)\end{array}$ & $\begin{array}{l}0.323 \\
(1.20)\end{array}$ \\
\hline In(FIRM_SIZE) & $\begin{array}{l}0.203^{\star * \star} \\
(3.55)\end{array}$ & $\begin{array}{l}0.211^{\star \star \star} \\
(3.69)\end{array}$ & $\begin{array}{l}0.133^{\star \star} \\
(2.20)\end{array}$ & $\begin{array}{l}0.140^{* *} \\
(2.26)\end{array}$ \\
\hline ANALYSTS & $\begin{array}{l}-0.012 \\
(-1.03)\end{array}$ & $\begin{array}{l}-0.014 \\
(-1.22)\end{array}$ & $\begin{array}{c}0.004 \\
(0.33)\end{array}$ & $\begin{array}{l}0.004 \\
(0.34)\end{array}$ \\
\hline VOLATILITY & $\begin{array}{l}0.280 \\
(0.28)\end{array}$ & $\begin{array}{r}0.237 \\
(0.26)\end{array}$ & $\begin{array}{l}-0.658 \\
(-0.88)\end{array}$ & $\begin{array}{l}-0.661 \\
(-0.99)\end{array}$ \\
\hline INST_OWN & $\begin{array}{l}-0.088 \\
(-0.30)\end{array}$ & $\begin{array}{l}-0.085 \\
(-0.22)\end{array}$ & $\begin{array}{l}-0.170 \\
(-0.86)\end{array}$ & $\begin{array}{l}-0.170 \\
(-0.83)\end{array}$ \\
\hline STAGGERED_BOARD & $\begin{array}{l}-0.222 \\
(-1.32)\end{array}$ & $\begin{array}{l}-0.222^{\star} \\
(-1.68)\end{array}$ & - & - \\
\hline PRIOR_RET & $\begin{array}{l}-0.142 \\
(-1.10)\end{array}$ & $\begin{array}{l}-0.166 \\
(-1.40)\end{array}$ & - & - \\
\hline $\begin{array}{l}F \text {-test } p \text {-value: } \beta_{1}+\beta_{2}=0 \\
F \text {-test } p \text {-value: } \beta_{2}+\beta_{3}=0\end{array}$ & $\begin{array}{l}0.000 \\
0.112\end{array}$ & $\begin{array}{l}0.000 \\
0.103\end{array}$ & $\begin{array}{l}0.000 \\
0.879\end{array}$ & $\begin{array}{l}0.000 \\
0.881\end{array}$ \\
\hline Industry and year dummies & Yes & Yes & Yes & Yes \\
\hline $\begin{array}{l}\text { No. of obs. } \\
\text { Pseudo- } R^{2}\end{array}$ & $\begin{array}{l}2,065 \\
0.335 \\
\end{array}$ & $\begin{array}{l}2,065 \\
0.301\end{array}$ & $\begin{array}{l}2,065 \\
0.083\end{array}$ & $\begin{array}{l}2,065 \\
0.082\end{array}$ \\
\hline
\end{tabular}

classify the misconduct to be severe if the CAR is in the bottom quartile or the settlement is in the top quartile. 
likelihood of both AC director turnover and losing board seats increases with the severity of the financial misconduct. However, even in cases of severe misconduct, the likelihood of turnover for highly $\mathrm{AC}$ connected directors is significantly lower than for less connected AC directors, and highly connected directors are less likely to lose additional board seats than less connected directors. Overall, the evidence in Tables 5 and 6 provides support for the hypothesis that AC directors with greater connectedness have fewer career concerns relative to their less connected counterparts. However, we also find evidence of ex post settling up in that highly connected AC directors appear to be held accountable in cases of misconduct, compared to non-AC directors.

\section{Robustness Tests}

\section{A. Firm Fixed Effects}

In our main tests, we control for year and industry fixed effects to mitigate time and industry-level omitted variable biases. We do not use firm-level fixed effects for several reasons. First, Greene (2004) cautions about applying fixed effects in non-linear models due to a finite sample bias when $t$ is less than 3; our unbalanced panel data have an average $t$ longer than 3 years, however, which should decrease this concern. Another concern is that there may be insufficient time-series variation in our connectedness measures to include firm-level fixed effects. However, we find significant variation. Specifically, the 25th (75th) percentile change in AC connectedness is $-8.4 \%$ (2.7\%). The 10th (90th) percentile change is $-73 \%(31 \%)$. In addition, of the 5,839 firms in our sample, 1,375 $(2,026)$ change from low AC connectedness to high AC connectedness (high AC connectedness to low AC connectedness) at least once over our sample period. Consequently, we revisit our main tests and control for firm fixed effects. We find similar results (untabulated) when including firm-level fixed effects.

\section{B. Director Accounting Expertise and Connectedness}

Regulatory reforms enacted as part of SOX call for ACs with sufficient financial expertise to ensure that members possess the knowledge to detect financial reporting misconduct. Using the SEC's narrow definition of accounting expertise, that is, individuals who have education and/or experience in accounting or auditing (e.g., auditor, CFO, controller, or accounting officer), we find that $25.7 \%$ of AC members are classified as accounting experts. In Table 7, we examine the joint effect of accounting expertise and director connectedness on financial reporting quality (Panel A), director turnover (Panel B), and future board seats (Panel C). In each model we include the controls previously identified in Tables 2 and 5, respectively.

The importance of the joint effect of accounting expertise and director connectedness is observed when we examine its effect on financial reporting quality in Panel A of Table 7. Specifically, we examine the percentage of AC members who are accounting experts (AC_ACCT_EXPERT), our dummy if AC connectedness is in the top quartile (HIGH_AC_CONNECT), and the interaction between these variables. Using the same three proxies for accruals reported in Table 2, we find that high AC connectedness continues to be associated with 
TABLE 7

Accounting Expertise and Director Connectedness

Table 7 examines the effect of accounting expertise on financial reporting quality, director turnover, future board seats, and financial reporting quality. Panel A examines financial reporting quality, Panel B examines the likelihood of director turnover, and Panel $\mathrm{C}$ examines future board seats. Control variables included in each model come from Table 2 for Panel A and Table 5 for Panels B and C. Variable definitions are in Appendices C and D, and details on the propensityscore-matched sample are in Table 2. The $t(z)$-statistics (in parentheses) are adjusted for heteroscedasticity using the Huber-White sandwich estimator and are corrected for clustering at the firm level. ${ }^{*},{ }^{* *}$, and ${ }^{* * *}$ indicate significance at the $10 \%, 5 \%$, and $1 \%$ levels using 2-tailed tests, respectively.

Panel A. Financial Reporting Quality

\begin{tabular}{|c|c|c|c|}
\hline \multirow[t]{2}{*}{ 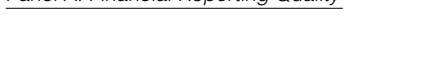 } & \multicolumn{3}{|c|}{ Dependent Variable } \\
\hline & $\mathrm{HN}$ & DGLS & $A Q$ \\
\hline Variables & 1 & 2 & 3 \\
\hline AC_ACCT_EXPERT & $\begin{array}{l}-0.005^{\star} \\
(-1.82)\end{array}$ & $\begin{array}{l}-0.005^{\star} \\
(-1.89)\end{array}$ & $\begin{array}{l}-0.006^{\star \star} \\
(-1.97)\end{array}$ \\
\hline HIGH_AC_CONNECT & $\begin{array}{l}-0.003^{\star \star} \\
(-2.26)\end{array}$ & $\begin{array}{l}-0.003^{\star \star} \\
(-2.29)\end{array}$ & $\begin{array}{l}-0.004^{\star \star} \\
(-2.33)\end{array}$ \\
\hline AC_ACCT_EXPERT $\times$ HIGH_AC_CONNECT & $\begin{array}{l}-0.003^{\star} \\
(-1.85)\end{array}$ & $\begin{array}{l}-0.002^{*} \\
(-1.92)\end{array}$ & $\begin{array}{l}-0.002^{\star} \\
(-1.80)\end{array}$ \\
\hline $\begin{array}{l}\text { Controls } \\
\text { Industry and year dummies }\end{array}$ & $\begin{array}{l}\text { Yes } \\
\text { Yes }\end{array}$ & $\begin{array}{l}\text { Yes } \\
\text { Yes }\end{array}$ & $\begin{array}{l}\text { Yes } \\
\text { Yes }\end{array}$ \\
\hline \multirow[t]{3}{*}{$\begin{array}{l}\text { No. of obs. } \\
\text { Adj./Pseudo- } R^{2}\end{array}$} & $\begin{array}{r}20,421 \\
0.153\end{array}$ & $\begin{array}{r}20,421 \\
0.115\end{array}$ & $\begin{array}{r}19,345 \\
0.122\end{array}$ \\
\hline & \multicolumn{3}{|c|}{ Sample } \\
\hline & Full & Matched & $\begin{array}{l}\text { Misconduct } \\
\text { Only }\end{array}$ \\
\hline Variables & 1 & 2 & 3 \\
\hline \multicolumn{4}{|l|}{ Panel B. Director Turnover } \\
\hline ACCT_EXPERT & $\begin{array}{l}-0.127 \\
(-1.23)\end{array}$ & $\begin{array}{l}-0.150 \\
(-1.20)\end{array}$ & $\begin{array}{l}-0.110 \\
(-1.10)\end{array}$ \\
\hline HIGH_D_CONNECT & $\begin{array}{l}-0.433^{\star \star \star} \\
(-18.00)\end{array}$ & $\begin{array}{l}-0.412^{\star \star} \\
(-2.28)\end{array}$ & $\begin{array}{l}-0.487^{\star \star \star} \\
(-2.76)\end{array}$ \\
\hline ACCT_EXPERT $\times$ HIGH_D_CONNECT & $\begin{array}{l}-0.130 \\
(-1.30)\end{array}$ & $\begin{array}{l}-0.169 \\
(-1.55)\end{array}$ & $\begin{array}{l}-0.129 \\
(-1.29)\end{array}$ \\
\hline $\begin{array}{l}\text { Controls } \\
\text { Industry and year dummies }\end{array}$ & $\begin{array}{l}\text { Yes } \\
\text { Yes }\end{array}$ & $\begin{array}{l}\text { Yes } \\
\text { Yes }\end{array}$ & $\begin{array}{l}\text { Yes } \\
\text { Yes }\end{array}$ \\
\hline $\begin{array}{l}\text { No. of obs. } \\
\text { Pseudo- } R^{2}\end{array}$ & $\begin{array}{r}164,915 \\
0.198\end{array}$ & $\begin{array}{l}3,712 \\
0.268\end{array}$ & $\begin{array}{l}2,065 \\
0.322\end{array}$ \\
\hline \multicolumn{4}{|l|}{$\underline{\text { Panel C. Future Boards }}$} \\
\hline ACCT_EXPERT & $\begin{array}{l}0.125^{\star} \\
(1.89)\end{array}$ & $\begin{array}{c}0.108 \\
(1.60)\end{array}$ & $\begin{array}{c}0.082 \\
(1.41)\end{array}$ \\
\hline HIGH_D_CONNECT & $\begin{array}{l}0.533^{\star \star \star} \\
(2.80)\end{array}$ & $\begin{array}{l}0.525^{\star \star \star} \\
(2.91)\end{array}$ & $\begin{array}{l}0.209^{\star \star} \\
(2.50)\end{array}$ \\
\hline ACCT_EXPERT $\times$ HIGH_D_CONNECT & $\begin{array}{c}0.151 \\
(1.30)\end{array}$ & $\begin{array}{l}0.150 \\
(1.55)\end{array}$ & $\begin{array}{c}0.172 \\
(1.45)\end{array}$ \\
\hline $\begin{array}{l}\text { Controls } \\
\text { Industry and year dummies }\end{array}$ & $\begin{array}{l}\text { Yes } \\
\text { Yes }\end{array}$ & $\begin{array}{l}\text { Yes } \\
\text { Yes }\end{array}$ & $\begin{array}{l}\text { Yes } \\
\text { Yes }\end{array}$ \\
\hline $\begin{array}{l}\text { No. of obs. } \\
\text { Pseudo- } R^{2}\end{array}$ & $\begin{array}{r}164,915 \\
0.035\end{array}$ & $\begin{array}{l}3,712 \\
0.063\end{array}$ & $\begin{array}{l}2,065 \\
0.082\end{array}$ \\
\hline
\end{tabular}

Dependent Variable

better financial reporting quality. Further, accounting expertise is weakly related to better reporting quality, as shown by the negative and significant coefficient on AC_ACCT_EXPERT in column 1 of Panel A. More important, we find that the interaction (AC_ACCT_EXPERT $\times$ HIGH_AC_CONNECT) is associated with better financial reporting quality for all three proxies for accounting quality measures. These results suggest that accounting expertise and AC connectedness complement each other; executives who are shielded from career concerns 
(i.e., highly connected members) and who are also accounting experts are better able to monitor the firm.

In Panels $\mathrm{B}$ and $\mathrm{C}$ of Table 7, we include a dummy for director accounting expertise (ACCT_EXPERT), a dummy for director connectedness (HIGH_D_ CONNECT), and their interaction to examine the combined effect of these two director characteristics on director career consequences. The negative and significant coefficients on HIGH_D_CONNECT support our prediction that wellconnected AC members are shielded from career concerns. The coefficient on accounting expertise indicates that after controlling for connectedness, accounting expertise has no effect on director turnover or future board seats. The interaction term (ACCT_EXPERT $\times$ HIGH_D_CONNECT) is also insignificant, suggesting that accounting expertise does not have additional predictive power over connectedness when it comes to the career concerns of directors. These results are consistent across our full, matched, and financial misconduct samples, reported in columns $1-3$, respectively.

\section{Corporate Governance and Director Connectedness}

In Section IV.D, we examine the labor market opportunities of connected $\mathrm{AC}$ directors following the detection of misconduct. Our evidence suggests that well-connected AC directors have fewer career concerns relative to less connected AC directors, but when compared to non-AC directors, connected AC directors are subject to ex post settling up in cases of misconduct. Prior studies find that stronger governance helps mitigate managerial opportunistic behavior and misconduct (Shleifer and Vishny (1997)). A firm's governance practices may also impact directors' career opportunities. Consequently, Table 8 reexamines the analysis in Table 5 after controlling for the strength of governance. In particular, we examine the impact of poor governance on the career consequences of highly connected AC directors (compared to less connected AC directors) following the detection of misconduct. ${ }^{29}$ We find that POOR_GOVERN has no significant predictive power in explaining either director turnover or future board seats. In addition, the interaction term (HIGH_D_CONNECT $\times$ POOR_GOVERN) is insignificant in all specifications, suggesting that governance does not mitigate the effect of director connectedness on their career consequences following firm misconduct.

\section{Alternative Measures of Director Connectedness}

Recall that our connectedness measure incorporates all 4 centrality metrics: degree, betweenness, closeness, and eigenvector. Since each measure examines a different, but highly correlated, aspect of the importance of an individual in the network, it may be that one connectedness variable has more predictive power over another. To test this prediction, we revisit our director turnover and future board seat analysis using each of the 4 centrality measures rather than overall

\footnotetext{
${ }^{29}$ As in Table 4, POOR_GOVERN is a dummy variable equal to 1 if the firm is in the top quartile of anti-takeover clauses, according to the Bebchuk et al. (2009) E-index. In untabulated results, we use the G-index and the measure in Dhaliwal, Naiker, and Navissi (2006) for AC governance strength and find similar results. In addition, we find no incremental effect of poor governance when comparing connected AC directors to connected non-AC directors.
} 


\section{Corporate Governance and Director Connectedness}

Table 8 examines the effect of corporate governance on director turnover and future board seats. POOR_GOVERN is a dummy variable that indicates the firm is in the top quartile in terms of antitakeover clauses according to the Bebchuk et al. (2009) E-index. Panel A examines the likelihood of director turnover and Panel B reports results on the directors' future board seats. Control variables included in each model and details on the propensity-score-matched sample are provided in Table 2. Variable definitions are provided in Appendix D. The $z(t)$-statistics (in parentheses) are adjusted for heteroscedasticity using the Huber-White sandwich estimator and are corrected for clustering at the firm level. ${ }^{*},{ }^{* *}$, and *** indicate significance at the $10 \%, 5 \%$, and $1 \%$ levels using 2 -tailed tests, respectively.

\begin{tabular}{|c|c|c|c|}
\hline \multirow{3}{*}{ Variables } & \multicolumn{3}{|c|}{ Sample } \\
\hline & Full & Matched & $\begin{array}{l}\text { Misconduct } \\
\text { Only }\end{array}$ \\
\hline & 1 & 2 & 3 \\
\hline \multicolumn{4}{|l|}{ Panel A. Director Turnover } \\
\hline POOR_GOVERN & $\begin{array}{r}0.025 \\
(1.29)\end{array}$ & $\begin{array}{l}0.029 \\
(0.70)\end{array}$ & $\begin{array}{r}0.085 \\
(1.27)\end{array}$ \\
\hline HIGH_D_CONNECT & $\begin{array}{l}-0.378^{\star \star *} \\
(-13.67)\end{array}$ & $\begin{array}{l}-0.402^{\star \star} \\
(-2.22)\end{array}$ & $\begin{array}{l}-0.423^{\star \star \star} \\
(-2.68)\end{array}$ \\
\hline HIGH_D_CONNECT × POOR_GOVERN & $\begin{array}{l}-0.026 \\
(-1.32)\end{array}$ & $\begin{array}{l}-0.095 \\
(-0.90)\end{array}$ & $\begin{array}{l}-0.786 \\
(-1.33)\end{array}$ \\
\hline $\begin{array}{l}\text { Controls } \\
\text { Industry and year dummies }\end{array}$ & $\begin{array}{l}\text { Yes } \\
\text { Yes }\end{array}$ & $\begin{array}{l}\text { Yes } \\
\text { Yes }\end{array}$ & $\begin{array}{l}\text { Yes } \\
\text { Yes }\end{array}$ \\
\hline $\begin{array}{l}\text { No. of obs. } \\
\text { Pseudo- } R^{2}\end{array}$ & $\begin{array}{r}21,871 \\
0.168\end{array}$ & $\begin{array}{l}2,638 \\
0.213\end{array}$ & $\begin{array}{l}1,225 \\
0.253\end{array}$ \\
\hline \multicolumn{4}{|l|}{$\underline{\text { Panel B. Future Boards }}$} \\
\hline POOR_GOVERN & $\begin{array}{c}0.022 \\
(1.46)\end{array}$ & $\begin{array}{l}0.119^{*} \\
(1.75)\end{array}$ & $\begin{array}{l}0.182^{\star \star} \\
(2.11)\end{array}$ \\
\hline HIGH_D_CONNECT & $\begin{array}{l}0.460^{* \star \star} \\
(2.63)\end{array}$ & $\begin{array}{l}0.473^{\star \star \star} \\
(2.69)\end{array}$ & $\begin{array}{l}0.201^{\star \star} \\
(2.33)\end{array}$ \\
\hline HIGH_D_CONNECT $\times$ POOR_GOVERN & $\begin{array}{l}-0.289 \\
(-0.89)\end{array}$ & $\begin{array}{l}-0.566 \\
(-1.02)\end{array}$ & $\begin{array}{l}-0.690 \\
(-1.16)\end{array}$ \\
\hline $\begin{array}{l}\text { Controls } \\
\text { Industry and year dummies }\end{array}$ & $\begin{array}{l}\text { Yes } \\
\text { Yes }\end{array}$ & $\begin{array}{l}\text { Yes } \\
\text { Yes }\end{array}$ & $\begin{array}{l}\text { Yes } \\
\text { Yes }\end{array}$ \\
\hline $\begin{array}{l}\text { No. of obs. } \\
\text { Pseudo- } R^{2}\end{array}$ & $\begin{array}{r}21,871 \\
0.032\end{array}$ & $\begin{array}{l}2,638 \\
0.055\end{array}$ & $\begin{array}{l}1,225 \\
0.074\end{array}$ \\
\hline
\end{tabular}

connectedness. Untabulated results show that each measure has similar predictive power in our regressions, which is not surprising given the high correlations across each measure (see Panel B of Table B2). Further, when all 4 metrics are used simultaneously, degree is insignificant, whereas the other three remain significant at the $10 \%$ level.

We examine several other measures of connectedness. In our main tests, we control for other director characteristics in the regressions. Alternatively, when we develop our AC and board connectedness measures, we first orthogonalize our director-level connectedness measure with the proxies for director ability: director age, education, and the number of board seats. Orthogonalization also mitigates the concern that director connectedness may increase mechanically over time as additional connections accumulate. We obtain similar results using the orthogonalized measure. Second, rather than averaging individual connectedness values at the board/committee level in each year, we aggregate them at the board/committee level. The advantage of our average measure is that the aggregate value is positively correlated with board/committee size. However, the average and aggregate metrics have a correlation of 0.68 and provide similar results. Finally, we follow Larcker et al. (2013) and rank directors into quintiles by degree, 
closeness, betweenness, and eigenvector centrality, and use the average ranking to measure overall connectedness. The correlation between our factor score and Larcker et al.'s " $N$-score" is 0.69 . Our findings are robust to the use of the $N$-score approach.

\section{E. Alternative Measures of Financial Misconduct}

Karpoff, Koester, Lee, and Martin (2017) examine 4 popular databases that identify restatements, securities class action lawsuits, and AAERs, and show that the results of empirical tests can depend on the database used. Our original tests use the Audit Analytics Non-Compliance database to identify earnings restatements (one of the databases examined in Karpoff et al.) and the Legal Case database to identify financial misconduct including restatement-, fraud-, and financial-reporting-related lawsuits (which Karpoff et al. do not examine). For robustness, we revisit our misconduct analyses using 2 other data sources discussed in Karpoff et al. The first is the U.S. Government Accountability Office (GAO) database, which identifies 2,705 restatement announcements from Jan. 1, 1997 through June 30, 2006. The second is based on the SEC AAER database, which we obtain from the Dechow data library (http://accounting.haas.berkeley.edu/ cfrm/aaer-dataset.html/index.html). Using these alternative data sources, we obtain quantitatively and qualitatively similar results. We conclude that the data biases outlined in Karpoff et al. do not affect our main findings.

\section{Conclusion}

We provide evidence of a specific channel through which director connectedness improves monitoring in the firm: financial reporting quality. Using various measures of abnormal accruals and accounting conservatism, we find that the connectedness of independent, non-co-opted AC members has a positive effect both on the quality of financial reporting and on accounting conservatism. The same effect is not seen when examining non-AC members or co-opted AC members, indicating that directors who are completely independent of the CEO and are members of certain committees are more instrumental in certain aspects of monitoring. Our results are robust to an examination of exogenous changes in board structure caused by member deaths. We further find that our results are stronger in small firms and firms with less busy AC directors, indicating that self-selection is not driving our results.

We also examine the market reaction to the deaths of AC members and find that the loss of a highly connected director is associated with a $3 \%$ lower announcement return than the loss of a less connected director. The observed negative announcement returns associated with the death of a well-connected director is exacerbated for firms with an otherwise less connected ACs and for firms with weaker internal governance structures. Although regulators have been pushing for greater board independence, our evidence suggests that the social connectedness of directors is also important in monitoring the firm and protecting shareholders' interests.

Finally, we examine the effect of connectedness on directors' career paths. When we restrict our sample to members of the AC, we find that well-connected 
AC members are less likely to experience turnover and more likely to gain additional boards seats in the aftermath of misconduct when compared to AC members who are not well connected. However, Srinivasan (2005) finds that AC directors are more likely than non-AC directors to experience turnover following the detection of financial misconduct. Consequently, we also compare the career consequences of well-connected AC members to those of well-connected nonAC members. Holding director connectedness constant, the results are consistent with ex post settling up: Highly connected AC directors at firms that experience financial misconduct are more likely to face turnover and less likely to gain future board seats than highly connected non-AC directors. Overall, our results suggest that although connectedness improves the career prospects of $\mathrm{AC}$ directors even in the event of misconduct, connected AC directors pay a price relative to connected non-AC directors following financial misconduct.

\section{Appendix A. Centrality Measures}

Newman (2003) notes that it is important to address the impact of an individual in the entire network (i.e., identify which individuals are most connected to others or have the most influence). Consequently, our connectedness measure includes 4 centrality measures developed in network theory that capture not only social ties but, more important, the quality of those connections: degree, betweenness, closeness, and eigenvector centrality, as introduced in Proctor and Loomis (1951), Freeman (1977), Sabidussi (1966), and Bonacich (1972), respectively. Although there is no theory as to which measure is superior, each captures distinct aspects of the relative importance of every individual in the entire network.

Degree captures the number of direct links an individual has with other individuals in the network. The more connections the individual has, the more important she is in the network. The average number of connections of the directors on the board/committee is our board/committee-level measure of degree. Although simple to construct, degree does not incorporate sources of information obtained through indirect links.

Betweenness represents the importance of an individual serving as the shortest information bridge or intermediary for other members (Freeman (1977)). Individuals with a higher betweenness measure have access to richer and more differentiated information. Betweenness is the sum of the shortest paths between all pairs of other individuals that pass through a person, scaled by the total number of shortest paths between the same pair of individuals. We standardize the measure by $(n-1)(n-2) / 2$, where $n$ is the number of members.

Closeness measures how quickly directors can access other directors in the network. It is defined as the inverse of the sum of an individual's distances to all other members (Sabidussi (1966)). The closer an individual is, the lower her total distance to all other members. Intuitively, having closer connections to more people makes information exchange quicker and more readily available, thus resulting in greater influence on others and higher social power.

Eigenvector centrality is based on the notion that not all individuals connected to a given person are equally important. Essentially, it is a weighted degree measure, with the weights based on how well connected each direct link is. Google's PageRank is a variant of this measure, which takes into account the relative importance or popularity of connected Web pages. A person who is connected with more important individuals is herself more important in the information dissemination channel and will have higher eigenvector centrality, all else equal. We use the Perron-Frobenius theorem to ensure that all 
eigenvectors are positive and that only the greatest eigenvalue results in the desired centrality measure.

Suppose there are $N$ directors (nodes) in the entire network and the connections between them. We define their degree, betweenness, closeness, and eigenvector centrality as follows:

1) Degree: Let the number of connections that director $A$ has with all the other directors, denoted as $C_{D}\left(A^{\prime}\right)$. A director's degree centrality is defined as $C_{D}(A)=C_{D}\left(A^{\prime}\right) /(N-1)$.

2) Let the director $C$ 's betweenness

$$
C_{B}\left(C^{\prime}\right)=\sum_{A<B} \frac{m(A, B ; C)}{m(A, B)},
$$

where $m(A, B ; C)$ is the number of shortest paths between directors $A$ and $B$ through director $C$, and $m(A, B)$ is the number of shortest paths between $A$ and $B$. The director's betweenness is defined as

$$
C_{B}(C)=\frac{C_{B}\left(C^{\prime}\right)}{(N-1)(N-2) / 2} .
$$

3) Let director $A$ 's absolute closeness be

$$
C_{C}\left(A^{\prime}\right)=\frac{1}{\sum_{A \in N} d(A, B)},
$$

where $N$ represents the entire network, and $d(A, B)$ is the number of connections in a shortest path connecting directors $A$ and $B$. Directors A's closeness is defined as

$$
C_{C}(A)=(N-1) \times C_{C}\left(A^{\prime}\right) .
$$

4) Suppose the $m \times m$ matrix $M$ is the adjacency matrix of the network, that is, $M_{i j}=1$ if there is a link between director/node $i$ and director/node $j$, and $M_{i j}=0$ otherwise. Also suppose the $m \times 1$ vector $p$ satisfies the following two conditions: i) $M p=a p$, where $a$ is the largest eigenvalue of $M$, and ii) $\max _{i}\left(p_{i}\right)=1$. Eigenvector centrality of director $A$ $C_{E}(A)$ is defined as the $A$ th element of $p$.

Not every pair of directors in the network is connected directly. In this case, multiple components of the network occur. Each component is defined as a subset of directors that can be reached from one another by connections between them indirectly. Betweenness and closeness as defined above are not calculable if the network has multiple components. Following the approach by Sabidussi (1966), we solve this problem by first calculating $C_{B}(A)$ and $C_{C}(A)$ over the component to which director $A$ belongs, and then scaling them by the ratio of the size of this component over $N$.

\section{Appendix B. An Illustration of Director Network}

Appendix B provides an illustration of a simple director network (Figure B1). We begin by explaining the calculation of each of the 4 centrality measures for this network and summarize the measures for each director within the network in Table B1. Table B2 then provides summary statistics of the director centrality measures for the actual sample used in this article.

This simple network has 10 directors. Each director is connected with every other director either directly or indirectly. We describe the calculations of the 4 centrality metrics 
FIGURE B1

An Illustration of Director Network

Figure B1 represents a hypothetical network. It serves as a simple example to demonstrate the distinction between degree, closeness, and betweenness measures.

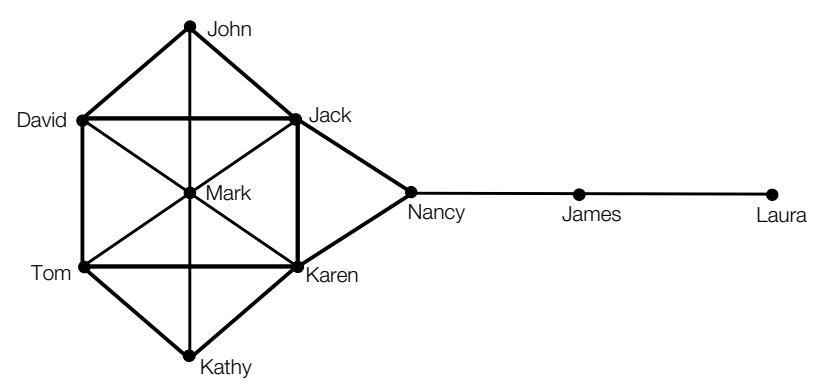

TABLE B1

Summary of Centrality Measures of the Directors in the Network

Table B1 summarizes the 4 centrality measures for each of the directors in Figure B1. The calculation of the 4 centrality measures is described in Appendix A.

\begin{tabular}{|c|c|c|c|c|}
\hline$\underline{\text { Director }}$ & Degree & Betweenness & Closeness & Eigenvector \\
\hline David & 0.444 & 0.023 & 0.529 & 0.732 \\
\hline Tom & 0.444 & 0.023 & 0.529 & 0.732 \\
\hline John & 0.333 & 0.000 & 0.500 & 0.594 \\
\hline Mark & 0.667 & 0.102 & 0.600 & 1.000 \\
\hline Kathy & 0.333 & 0.000 & 0.500 & 0.594 \\
\hline Jack & 0.556 & 0.231 & 0.643 & 0.827 \\
\hline Karen & 0.556 & 0.231 & 0.643 & 0.827 \\
\hline Nancy & 0.333 & 0.389 & 0.600 & 0.407 \\
\hline James & 0.222 & 0.222 & 0.429 & 0.100 \\
\hline Laura & 0.111 & 0.000 & 0.310 & 0.023 \\
\hline
\end{tabular}

for representative directors. The measures for all directors are summarized in Table B1. Mark has the highest degree centrality because he is directly connected to 6 other directors. His degree metric is calculated as $6 /(10-1)=0.667$. However, his closeness metric is not the highest because it takes 4 steps for him to reach Laura, 3 steps to James, 2 steps to Nancy, and 1 step to the rest of the directors. As such, his closeness centrality is $(10-1) \times$ $(1 /(4+3+2+1 \times 6))=0.6$. Jack and Karen have the highest closeness measure as they can quickly reach other members of the network, considering all direct and indirect links.

Betweenness estimates the shortest path between all other pairs of the network. For example, there are 36 pairs of other directors in the network and David is 1 of the 2 shortest paths between Tom and John. Also, David lies on 1 of the 3 shortest paths between Tom and Jack. No other shortest path passes through him. Therefore, we calculate his betweenness as $(1 / 2+1 / 3) / 36=0.023$. By this logic, we find that Nancy actually has the highest betweenness metric. In other words, she serves as a crucial information flow broker of the network.

Closeness is the inverse of the sum of an individual's distances to all other members. Take John, for example. Counting his connections with all the other members and summing them up yields $(1+2+1+2+1+2+2+3+4)=18$. The total number of nodes minus 1 in this case is $10-1=9$. John's closeness is thus $9 / 18=0.5$.

Eigenvector centrality is a weighted sum of the degree measure. John and Nancy have the same degree metric (i.e., 0.333). However, John connects to David, Mark, and Jack, 
TABLE B2

Director Centrality Summary Statistics

Table B2 presents the summary statistics of director level centrality measures. DEGREE and BETWEENNESS are multiplied by $10^{4}$ and CLOSENESS and EIGENVECTOR are multiplied by $10^{2}$.

Panel A. Director-Level Centrality Measures

\begin{tabular}{|c|c|c|c|c|c|c|}
\hline Variable & Mean & Median & Std. Dev. & $\begin{array}{c}\text { Lower } \\
\text { Quartile }\end{array}$ & $\begin{array}{c}\text { Upper } \\
\text { Quartile } \\
\end{array}$ & Skew \\
\hline DEGREE & 3.031 & 2.446 & 3.665 & 0.529 & 5.215 & 3.228 \\
\hline CLOSENESS & 13.252 & 15.011 & 9.256 & 2.785 & 21.662 & -0.772 \\
\hline BETWEENNESS & 0.530 & 0.000 & 2.147 & 0.000 & 0.190 & 10.320 \\
\hline EIGENVECTOR & 1.256 & 0.117 & 4.753 & 0.020 & 0.585 & 8.073 \\
\hline
\end{tabular}

Panel B. Correlations between Centrality Measures

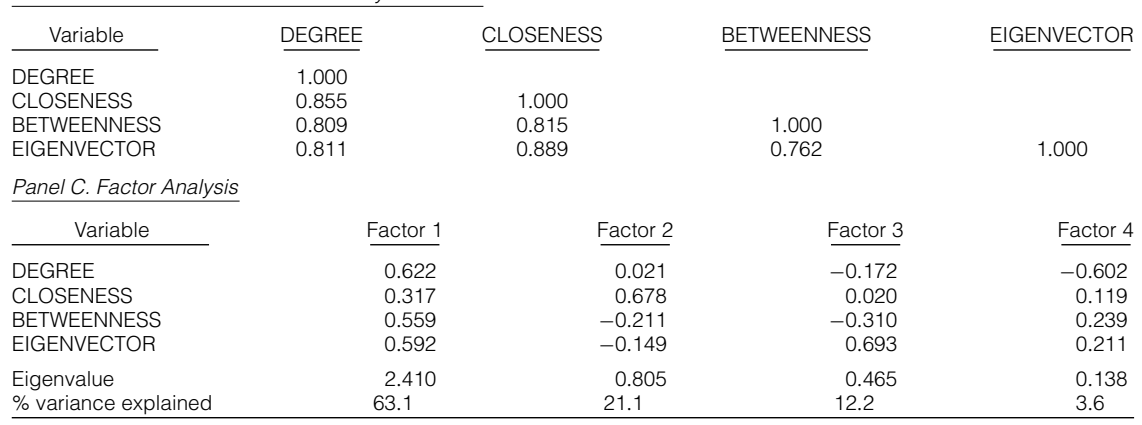

whereas Nancy connects to Jack, Karen, and James. Considering the degree measures of the 6 connected directors, we can see that i) David's degree is higher than James's and ii) Mark's degree is higher than Karen's. As such, John connects to directors with higher degree than Nancy does. The difference is reflected in the eigenvector measure: John's eigenvector centrality is 0.594 and Nancy's is 0.407 .

\section{Appendix C. Financial Reporting Quality and Conservatism Measures}

\section{HN: Unsigned Abnormal Accruals (Hribar and Nichols (2007))}

We first estimate the following regression for each year and Fama-French (1997) industry:

$$
\mathrm{TACC}=\alpha+\beta_{1} \Delta \mathrm{REV}+\beta_{2} \mathrm{PPE}+\xi,
$$

where TACC is total accruals, defined as income before extraordinary items minus cash from operations divided by lagged total assets. $\triangle \mathrm{REV}$ is the change in sales adjusted for the change in receivables, divided by lagged total assets. PPE is gross property, plant, and equipment, scaled by lagged total assets. We then calculate the abnormal accruals as the residual term in the regression, that is, TACC $-\left(\alpha+\beta_{1} \Delta \mathrm{REV}+\beta_{2} \mathrm{PPE}\right)$, and $\mathrm{HN}$ is the absolute value of the residual (abnormal accruals).

2. DGLS: Industry-Adjusted Absolute Value of DD Residual (Dechow et al. (2011))

We first regress working capital accruals (WC_ACC) on operating cash flows in the current year $\left(\mathrm{OCF}_{t}\right)$, the preceding year $\left(\mathrm{OCF}_{t-1}\right)$, and the following year $\left(\mathrm{OCF}_{t+1}\right)$ :

$$
\mathrm{WC} \_\mathrm{ACC}_{i, t}=\alpha_{0, i}+\beta_{1, i} \mathrm{OCF}_{i, t-1}+\beta_{2, i} \mathrm{OCF}_{i, t}+\beta_{3, i} \mathrm{OCF}_{i, t+1}+v_{i, t},
$$


where WC_ACC $=\Delta \mathrm{CA}-\Delta \mathrm{CL}-\Delta \mathrm{CASH}+\Delta \mathrm{STDEBT}+\Delta \mathrm{TAXES}$, where $\Delta \mathrm{CA}$ is change in current assets between years $t-1$ and $t, \Delta \mathrm{CL}$ is change in current liabilities between years $t-1$ and $t, \Delta \mathrm{CASH}$ is change in cash and short-term investments between years $t-1$ and $t, \triangle$ STDEBT is change in short-term debt between years $t-1$ and $t$, and $\triangle$ TAXES is change in taxes payable between years $t-1$ and $t$. All variables are scaled by average total assets and winsorized at the $1 \%$ and $99 \%$ levels. We estimate the equation by year for each of the 2-digit SIC industry groups. DGLS is the absolute value of each firm's residual less the average absolute value for the corresponding industry.

\section{AQ: Performance-Matched Discretional Accruals (Kothari et al. (2005))}

We estimate abnormal accruals for each firm-year and subtract the value from the discretionary accruals of the performance-matched firm. The modified Jones (1991) model of abnormal accruals model is estimated cross sectionally each year using all firm-year observations in the same Fama-French (1997) industry:

$$
\mathrm{TA}_{i, t}=\beta_{0}+\beta_{1}\left(1 / \operatorname{ASSETS}_{i, t-1}\right)+\beta_{2}\left(\Delta \mathrm{SALES}_{i, t}-\Delta \mathrm{AR}_{i, t}\right)+\beta_{3} \mathrm{PPE}_{i, t}+\xi_{i, t},
$$

where TA (total accruals) is the change in noncash current assets minus the change in current liabilities excluding the current portion of long-term debt, minus depreciation and amortization, scaled by lagged total assets; $\triangle \mathrm{SALES}_{i, t}$ is change in sales; $\Delta \mathrm{AR}_{i, t}$ is change in accounts receivable; and $\mathrm{PPE}_{i, t}$ is gross property, plant, and equipment, all scaled using lagged total assets, ASSETS ${ }_{i, t-1}$. We use total assets as the deflator to mitigate heteroscedasticity in the residuals.

Residuals from the annual cross-sectional industry regression model in the modified Jones (1991) model are used to measure estimated abnormal accruals. We then match each firm-year observation with another firm from the same Fama-French (1997) industry and year with the closest return on assets in the current year, $\mathrm{ROA}_{i, t}$ (net income divided by total assets). We define the AQ for firm $i$ in year $t$ as the abnormal accrual in year $t$ minus the performance-matched abnormal accrual for year $t$.

\section{CSCORE (Khan and Watts (2009))}

Khan and Watts (2009) develop a firm-specific estimation of the timeliness of bad news and document evidence consistent with conservatism increasing in the measure (CSCORE). We use annual cross-sectional regressions to obtain CSCORE following Ahmed and Duellman ((2013), p. 10).

\section{SKEW (Beatty et al. (2008))}

For each firm-year, we calculate SKEW as the negative of the difference in the skewness of earnings and the skewness of cash flows over the 3 years before the current year.

\section{Appendix D. Additional Variables of Interest}

\section{Director-Level Variables}

AC: Dummy variable equal to 1 if the director is an audit committee (AC) member.

ACCT_EXPERT: Dummy variable equal to 1 if the director is considered an expert, where experts are those who currently hold or have held the position of CFO, CPA/CFA, controller, comptroller, treasurer, or any other position that is related to financial reporting.

BOARD_SEATS: Number of external (additional) board seats for the director.

D_AGE: Age of the director. 
D_TENURE: Number of years the director is on the AC.

D_TURNOVER: Dummy variable equal to 1 if the director is dismissed from the board.

D_FEMALE: Dummy variable equal to 1 if the director is female.

FUTURE_BOARDS: Number of board seats the director obtains in the future 3 years.

HIGH_D_CONNECT: Dummy variable equal to 1 if the AC member connectedness is in the top quartile among all the AC directors.

HIGH_EDU: Dummy variable indicating whether the director has obtained a degree from a prominent undergraduate or master of business administration (MBA) program.

HIGH_POST: Number of high-level positions (defined as higher than vice president) the director has obtained during his career.

Audit-Committee-Level Variables

AC_AGE: Average age of non-co-opted AC members.

AC_ACCT_EXPERT: Percentage of AC members that are considered experts, where experts are those who currently hold or have held the position of CFO, CPA/CFA, controller, comptroller, treasurer, or any other position that is related to financial reporting.

AC_BOARD_SEATS: Number of external (additional) board seats for AC member.

AC_BUSY: Proportion of independent, non-co-opted AC members sitting on 4 or more public firms' boards.

AC_CONNECT: Average of factor score of independent, non-co-opted AC members; factor score is based on all directors' degree, betweenness, closeness, and eigenvector centrality.

AC_FEMALE_RATIO: Percentage of female members on the AC.

AC_HIGH_EDU: Proportion of non-co-opted AC members that have obtained degrees from prominent undergraduate or MBA programs.

AC_HIGH_POST: Average number of high-level positions (defined as higher than vice president) that the non-co-opted $\mathrm{AC}$ members have obtained during their career.

AC_INDEPENDENCE: Number of independent, non-co-opted AC members scaled by total number of AC members.

AC_OTHR_CONNECT: Average of factor score of independent, co-opted AC members; factor score is based on all directors' degree, betweenness, closeness, and eigenvector centrality.

AC_SIZE: Natural log of the total number of AC members.

AC_TENURE: Average number of years the AC member has been on the committee.

HIGH_AC_CONNECT: Dummy variable equal to 1 if the AC connectedness is in the top quartile of the sample firms.

LOW_AC_CONNECT: Dummy variable equal to 1 if the AC connectedness is in the bottom quartile of the sample firms.

NON_AC_CONNECT: Average of factor score of non-audit committee board members; factor score is based on all directors' degree, betweenness, closeness, and eigenvector centrality.

\section{CEO Variables}

CEO_CONNECT: Factor score based on CEOs' degree, betweenness, closeness, and eigenvector centrality. 
CEO_POWER: Calculated using factor analysis to extract the common underlying latent variable, using $\mathrm{CEO}$ tenure, $\mathrm{CEO}$ equity ownership, and $\mathrm{CEO} /$ chairman duality.

CEO/AC_OVERLAP: Percentage of independent, non-co-opted AC members that have either overlapping historical experience or an interlocking relationship with the CEO.

CEO/D_OVERLAP: Dummy variable for whether the non-co-opted AC member has either overlapping historical experience or an interlocking relationship with the CEO.

CEO_TENURE: Number of years the CEO has held the top post.

\section{Firm-Level Variables}

ANALYSTS: Natural log of the number of analysts following the firm during the year.

CAR: Cumulative abnormal returns during the $(-2,+2)$ window around the $\mathrm{AC}$ member death announcement date.

CASH: Cash scaled by book value of total assets.

CASHFLOW: Cash flow divided by book value of total assets.

FIRM_SIZE: Total assets (\$million); $\ln ($ FIRM_SIZE) is the natural log of total assets.

FSCORE: Predicted probability of firm committing fraud based on Dechow et al. ((2011), p. 61).

INST_OWN: Proportion of common equity owned by institutional investors.

LEVERAGE: Book value of long-term debt divided by total assets.

LIT_RISK: Estimated probability of litigation based on model 3 in Kim and Skinner (2012, p. 302), calculated as eSUE/( $1+\mathrm{eSUE})$, where $\mathrm{SUE}_{t}=-7.883+0.566 \times$ $\mathrm{FPS}_{t}+0.518 \times$ ASSETS $_{t-1}+0.982 \times$ SALES_GROWTH $_{t-1}+0.379 \times$ RETURN $_{t-1}-$ $0.108 \times$ RETURN_SKEWNESS $_{t-1}+25.635 \times$ RETURN_STD_DEV $_{t-1}+0.00007 \times$ TURNOVER $_{t-1}$. FPS is equal to 1 if the firm is in the biotech (SIC codes 2833-2836 and 8731-8734), computer (3570-3577 and 7370-7374), electronics (3600-3674), or retail (5200-5961) industry, and 0 otherwise; ASSETS is natural log of total assets; SALES_GROWTH is current-year sales less last-year sales scaled by beginning-of-current-year total assets; RETURN is market-adjusted 12-month stock return; RETURN_SKEWNESS is skewness of the firm's 12-month return; RETURN_STD_DEV is standard deviation of the firm's 12-month returns; and TURNOVER is daily trading volume accumulated over the fiscal year scaled by beginning of the year's shares outstanding (in thousands).

MTB: Market value of common equity plus book value of long-term debt divided by total assets.

MISCONDUCT: Dummy variable equal to 1 in the year the public becomes aware of any fraudulent activity committed by the firm, as evidenced by SEC/DOJ legal actions related to accounting malpractice, financial reporting, fraud, or AAER.

OPER_CYCLE: Days in account receivables + days in inventory.

POOR_GOVERN: Dummy variable indicating the firm is in the top quartile of the Bebchuk et al. (2009) E-index.

PRIOR_RET: Buy-and-hold return during the prior 3 years.

ROA: Earnings before extraordinary items divided by total assets.

S_MISCONDUCT: Dummy variable equal to 1 if the misconduct settlement amount is in the top quartile or if the announcement CAR is in the bottom quartile.

STAGGERED_BOARD: Dummy variable equal to 1 if the firm has a staggered board.

VOLATILITY: Standard deviation of stock return calculated over prior 60 months.

$\sigma(\mathrm{OCF})$ : Standard deviation of operating cash flow, measured over the previous 10 years. $\sigma$ (SALES): Standard deviation of sales, measured over the previous 10 years. 


\section{Appendix E. Matched-Sample Statistics for Sample Used in Table 5}

In Table E1, we demonstrate that our matching process yields a non-misconduct matched sample with similar characteristics to that of our misconduct sample. To create the matched sample, we use a propensity-score-matching approach on the 1,032 firms with detected misconduct. Firms are matched in the year before the detection of misconduct based on firm size, ROA, CEO tenure, CEO connectedness, volatility, institutional ownership, analyst following, AC size, AC independence, average AC director tenure, average AC director age, AC female ratio, the Dechow et al. (2011) fraud score (FSCORE), and industry and year controls. Using one-to-one matching with a caliper of $0.1 \%$ without replacement, we are able to match 928 firms with misconduct to firms without.

We match on the Dechow et al. (2011) FSCORE to mitigate the possibility that better connected directors choose to sit on the boards of firms that are less likely to engage in fraudulent activity. Dechow et al. ((2011), p. 61) use data on legal actions from the SEC's AAERs to develop a prediction model for accounting fraud. They suggest that the ratio of the predicted probabilities of misstatements from their model to the unconditional probability of misstatements can be used as a measure of fraud likelihood relative to a random firm. However, the unconditional probability is specific to their study period (1982-2005). Because our study period does not correspond to theirs, we use the predicted probabilities and sample distribution to demarcate situations of high and low likelihood of potential misstatements. In untabulated results, we also use litigation risk (defined in Appendix D) to proxy for the reputational concerns of directors and find results similar to FSCORE.

\section{TABLE E1}

\section{Matched-Sample Statistics}

Table E1 provides summary statistics of the characteristics on which we match in the matched sample in Table 5. See Appendix D for full variable definitions.

\begin{tabular}{|c|c|c|c|}
\hline Variable & $\begin{array}{l}\text { Firms With } \\
\text { Misconduct }\end{array}$ & $\begin{array}{c}\text { Firms Without } \\
\text { Misconduct } \\
\end{array}$ & $t$-Test \\
\hline FIRM_SIZE (\$millions) & $4,035.962$ & $3,651.889$ & 1.35 \\
\hline $\mathrm{ROA}$ & 0.041 & 0.049 & 1.55 \\
\hline FSCORE & 1.234 & 1.188 & 1.21 \\
\hline INST_OWN & 0.682 & 0.670 & 1.62 \\
\hline ANALYSTS & 14.726 & 14.422 & 0.92 \\
\hline VOLATILITY & 0.038 & 0.050 & 1.23 \\
\hline CEO_TENURE & 7.530 & 8.702 & 1.09 \\
\hline CEO_CONNECT & 0.351 & 0.334 & 0.28 \\
\hline AC_SIZE & 3.462 & 3.480 & 0.40 \\
\hline AC_INDEPENDENCE & 0.705 & 0.710 & 1.50 \\
\hline AC_TENURE & 7.500 & 7.782 & 1.37 \\
\hline AC_AGE & 59.115 & 59.880 & 1.27 \\
\hline AC_FEMALE_RATIO & 0.106 & 0.100 & 0.50 \\
\hline
\end{tabular}

\section{References}

Abadie, A., and G. Imbens. "Large Sample Properties of Matching Estimators for Average Treatment Effects." Econometrica, 74 (2006), 235-267.

Adams, R. B., and D. Ferreira. "A Theory of Friendly Boards." Journal of Finance, 62 (2007), $217-250$.

Ahmed, A. S., and S. Duellman. "Managerial Overconfidence and Accounting Conservatism." Journal of Accounting Research, 51 (2013), 1-30.

Ai, C., and E. Norton. "Interaction Terms in Logit and Probit Models." Economics Letters, 80 (2003), 123-129.

Alam, Z.; M. Chen; C. Ciccotello; and H. Ryan. "Does the Location of Directors Matter? Information Acquisition and Board Decisions." Journal of Financial and Quantitative Analysis, 49 (2014), $131-164$. 
Anderson, R. C.; S. Mansi; and D. Reeb. "Board Characteristics, Accounting Report Integrity, and the Cost of Debt." Journal of Accounting and Economics, 37 (2004), 315-342.

Armstrong, C., and D. Larcker. "Discussion of the Impact of the Options Backdating Scandal on Shareholders and Taxes and the Backdating of Stock Option Exercise Date." Journal of Accounting and Economics, 47 (2009), 50-58.

Ashbaugh, H. A.; R. LaFond; and B. W. Mayhew. "Do Nonaudit Services Compromise Auditor Independence? Further Evidence.” Accounting Review, 78 (2003), 611-639.

Barnea, A., and I. Guedj. "Director Networks." Working Paper, HEC Montreal and Bates White, LLC (2014).

Basu, S. "The Conservatism Principle and the Asymmetric Timeliness of Earnings." Journal of Accounting and Economics, 24 (1997), 3-37.

Bates, T. W.; D. A. Becher; and J. I. Wilson. "Performance-Based Turnover on Corporate Boards." Working Paper, Arizona State University, Drexel University, and Indiana University. Available at http://ssrn.com/abstract=2654375 (2017).

Beatty, A.; J. Weber; and J. J. Yu. "Conservatism and Debt." Journal of Accounting and Economics, 45 (2008), 154-174.

Bebchuk, L.; A. Cohen; and A. Ferrell. "What Matters in Corporate Governance?” Review of Financial Studies, 22 (2009), 783-827.

Bédard, J.; S. M. Chtourou; and L. Courteau. "The Effect of Audit Committee Expertise, Independence, and Activity on Aggressive Earnings Management." Auditing: A Journal of Practice \& Theory, 23 (2004), 15-37.

Belliveau, M. A.; C. A. O'Reilly; and J. B. Wade. "Social Capital at the Top: Effects of Social Similarity and Status on CEO Compensation." Academy of Management Journal, 39 (1996), 1568-1593.

Bhagat, S., and B. S. Black. "The Uncertain Relationship between Board Composition and Firm Performance." Business Lawyer, 54 (1999), 921-963.

Bizjak, J. M.; M. L. Lemmon; and R. J. Whitby. "Option Backdating and Board Interlocks.” Review of Financial Studies, 22 (2009), 4821-4847.

Bonacich, P. "Factoring and Weighting Approaches to Status Scores and Clique Identification." Journal of Mathematical Sociology, 2 (1972), 113-120.

Brown, R.; N. Gao; E. Lee; and K. Stathopoulos. "What Are Friends for? CEO Networks, Pay and Corporate Governance." In Corporate Governance: Recent Developments and New Trends, S. Boubaker, B. D. Nguyen, and D. K. Nguyen, eds. Berlin, Germany: Springer Berlin Heidelberg (2012), 287-307.

Carcello, J.; T. Neal; Z. V. Palmrose; and S. Scholz. "CEO Involvement in Selecting Board Members, Audit Committee Effectiveness, and Restatements." Contemporary Accounting Research, 28 (2011), 396-430.

Cashman, G. D.; S. L. Gillan; and R. J. Whitby. "Expertise, Connections, and the Labor Market for Corporate Directors." Working Paper, Texas Tech University, University of Georgia, and Utah State University (2010).

Chemmanur, T., and I. Paeglis. "Management Quality, Certification, and Initial Public Offering." Journal of Financial Economics, 76 (2005), 331-368.

Chidambaran, N. K.; S. Kedia; and N. R. Prabhala. "CEO-Director Connections and Corporate Fraud." Working Paper, Fordham University, Rutgers Business School, and University of Maryland. Available at http://ssrn.com/abstract=2023030 (2012).

Chiu, P.-C.; S. H. Teoh; and F. Tian. "Board Interlocks and Earnings Management Contagion." Accounting Review, 88 (2013), 915-944.

Cohen, A., and C. C. Y. Wang. "How Do Staggered Boards Affect Shareholder Value? Evidence from a Natural Experiment." Journal of Financial Economics, 110 (2013), 627-641.

Cohen, L.; A. Frazzini; and C. Malloy. "Hiring Cheerleaders: Board Appointments of 'Independent' Directors.” Management Science, 58 (2012), 1039-1058.

Coles, J. L.; N. D. Daniel; and L. Naveen. "Co-Opted Boards.” Review of Financial Studies, 27 (2014), 1751-1796.

Coles, J. L., and C. K. Hoi. "New Evidence on the Market for Directors: Board Membership and Pennsylvania Senate Bill 1310.” Journal of Finance, 58 (2003), 197-230.

Coles, J. L.; A. Y. Wang; and C. Zhu. "Director Network and CEO Turnover." Working Paper, University of Utah, Auburn University, and New York University (2015).

Dechow, P. M., and I. D. Dichev. "The Quality of Accruals and Earnings: The Role of Accrual Estimation Errors." Accounting Review, 77 (2002), 35-59.

Dechow, P. M.; W. Ge; C. R. Larson; and R. G. Sloan. "Predicting Material Accounting Misstatements." Contemporary Accounting Research, 28 (2011), 17-82. 
Dechow, P. M.; W. Ge; and C. Schrand. "Understanding Earnings Quality: A Review of the Proxies, Their Determinants and Their Consequences." Journal of Accounting and Economics, 50 (2010), 344-401.

DeFond, M. L.; R. N. Hann; and X. Hu. "Does the Market Value Financial Expertise on Audit Committees of Boards of Directors?” Journal of Accounting Research, 43 (2005), 153-193.

Denis, D. K.; J. Lee; and K. J. Lee. "Director Connections, Board Appointments, and Director Reputation." Working Paper, University of Pittsburgh, University of Florida, and U.S. Securities and Exchange Commission (2014).

Dhaliwal, D. S.; V. Naiker; and F. Navissi. "Audit Committee Financial Expertise, Corporate Governance and Accruals Quality: An Empirical Analysis.” Working Paper, University of Arizona, The University of Melbourne, and Monash University. Available at http://ssrn.com/abstract=9066 90 (2006)

Dhaliwal, D. S.; V. Naiker; and F. Navissi. "The Association between Accruals Quality and the Characteristics of Accounting Experts and Mix of Expertise on Audit Committees." Contemporary Accounting Research, 27 (2010), 787-827.

Doyle, J.; W. Ge; and S. McVay. "Determinants of Weaknesses in Internal Control over Financial Reporting." Journal of Accounting and Economics, 44 (2007), 193-223.

Dyck, I. J. A.; A. Morse; and L. Zingales. "Who Blows the Whistle on Corporate Fraud?" Journal of Finance, 65 (2010), 2213-2253.

El-Khatib, R.; K. Fogel; and T. Jandik. "CEO Network Centrality and Merger Performance.” Journal of Financial Economics, 116 (2015), 349-382.

Engelberg, J.; P. Gao; and C. A. Parsons. "Friends with Money." Journal of Financial Economics, 103 (2012), 169-188.

Engelberg, J.; P. Gao; and C. A. Parsons. "The Price of a CEO's Rolodex.” Review of Financial Studies, 26 (2013), 79-114.

Fahlenbrach, R.; A. Low; and R. Stulz. "Do Independent Director Departures Predict Future Bad Events?" Review of Financial Studies, 30 (2017), 2313-2358.

Fama, E. F. "Agency Problems and the Theory of the Firm.” Journal of Political Economy, 88 (1980), 288-307.

Fama, E. F., and K. R. French. "Industry Costs of Equity.” Journal of Financial Economics, 43 (1997), $153-193$.

Fama, E. F., and M. C. Jensen. "Separation of Ownership and Control." Journal of Law and Economics, 26 (1983), 301-325.

Ferreira, M., and P. Laux. "Corporate Boards and SEOs: The Effect of Certification and Monitoring." Journal of Financial and Quantitative Analysis, 51 (2016), 899-927.

Fich, E. M., and A. Shivdasani. “Are Busy Boards Effective Monitors?” Journal of Finance, 61 (2006), 689-724.

Fich, E. M., and A. Shivdasani. "Financial Fraud, Director Reputation, and Shareholder Wealth." Journal of Financial Economics, 86 (2007), 306-336.

Fracassi, C. "Corporate Finance Policies and Social Networks.” Management Science, 63 (2017), 2420-2438.

Fracassi, C., and G. Tate. "External Networking and Internal Firm Governance." Journal of Finance, 67 (2012), 153-194.

Freeman, L. C. "A Set of Measures of Centrality Based on Betweenness." Sociometry, 40 (1977), $35-41$.

Gilson, S. "Bankruptcy, Boards, Banks, and Blockholders: Evidence on Changes in Corporate Ownership and Control When a Firms Default." Journal of Financial Economics, 27 (1990), 355-387.

Givoly, D., and C. Hayn. "The Changing Time-Series Properties of Earnings, Cash Flows and Accruals: Has Financial Reporting Become More Conservative?" Journal of Accounting and Economics, 29 (2000), 287-320.

Gompers, P. A.; J. L. Ishii; and A. Metrick. "Corporate Governance and Equity Prices.” Quarterly Journal of Economics, 11 (2003), 107-155.

Gormley, T. A., and D. A. Matsa. "Common Errors: How To (and Not To) Control for Unobserved Heterogeneity.” Review of Financial Studies, 27 (2014), 617-661.

Greene, W. H. "The Behaviour of the Maximum Likelihood Estimator of Limited Dependent Variable Models in the Presence of Fixed Effects.” Econometrics Journal, 7 (2004), 98-119.

Greene, W. H. "Testing Hypotheses about Interaction Terms in Nonlinear Models.” Economic Letters, 107 (2010), 291-296.

Harford, J. “Takeover Bids and Target Directors' Incentives: The Impact of a Bid on Directors' Wealth and Board Seats." Journal of Financial Economics, 69 (2003), 51-83. 
Harford, J., and R. Schonlau. "Does the Director Labor Market Offer Ex Post Settling-Up for CEOs? The Case of Acquisitions." Journal of Financial Economics, 110 (2013), 18-36.

He, J., and Z. Huang. "Board Informal Hierarchy and Firm Financial Performance: Exploring a Tacit Structure Guiding Boardroom Interactions.” Academy of Management Journal, 54 (2011), 1119-1139.

Healy, P. M. "The Effect of Bonus Schemes on Accounting Decisions." Journal of Accounting and Economics, 7 (1985), 85-107.

Hermalin, B. E., and M. S. Weisbach. "Endogenously Chosen Boards of Directors and Their Monitoring of the CEO.” American Economic Review, 88 (1998), 96-118.

Holthausen, R., and R. Watts. "The Relevance of Value Relevance Literature for Financial Accounting Standard Setting." Journal of Accounting and Economics, 31 (2001), 3-75.

Hooghiemstra, R., and J. van Manen. "The Independence Paradox: (Im)possibilities Facing Non-Executive Directors in the Netherlands." Corporate Governance: An International Review, 12 (2004), 314-324.

Hribar, P., and D. C. Nichols. "The Use of Unsigned Earnings Quality Measures in Tests of Earnings Management." Journal of Accounting Research, 45 (2007), 1017-1053.

Hwang, B. H., and S. Kim. "It Pays to Have Friends.” Journal of Financial Economics, 93 (2009), $138-158$.

Jones, J. "Earnings Management during Import Relief Investigations." Journal of Accounting Research, 29 (1991), 193-228.

Karpoff, J.; A. Koester; D. S. Lee; and G. S. Martin. "Proxies and Databases in Financial Misconduct Research.” Accounting Review, 92 (2017), 129-163.

Khan, M., and R. L. Watts. "Estimation and Empirical Properties of a Firm-Year Measure of Accounting Conservatism.” Journal of Accounting and Economics, 48 (2009), 132-150.

Khanna, V.; E. H. Kim; and Y. Lu. "CEO Connectedness and Corporate Fraud.” Journal of Finance, 70 (2015), 1203-1252.

Kim, I., and D. J. Skinner. "Measuring Securities Litigation Risk." Journal of Accounting and Economics, 53 (2012), 290-310.

Klein, A. "Audit Committee, Board of Director Characteristics, and Earnings Management." Journal of Accounting and Economics, 33 (2002), 375-400.

Knyazeva, A.; D. Knyazeva; and R. W. Masulis. "The Supply of Corporate Directors and Board Independence." Review of Financial Studies, 26 (2013), 1561-1605.

Kothari, S. P.; A. J. Leone; and C. E. Wasley. "Performance Matched Discretionary Accrual Measures." Journal of Accounting and Economics, 39 (2005), 163-197.

Krishnan, G. V., and G. Visvanathan. "Does the SOX Definition of an Accounting Expert Matter? The Association between Audit Committee Directors' Accounting Expertise and Accounting Conservatism." Contemporary Accounting Research, 25 (2008), 827-858.

Krishnan, J.; Y. Wen; and W. Zhao. "Legal Expertise on Corporate Audit Committees and Financial Reporting Quality." Accounting Review, 86 (2011), 2099-2130.

Larcker, D. F.; E. C. So; and C. C. Y. Wang. "Boardroom Centrality and Firm Performance." Journal of Accounting and Economics, 55 (2013), 225-250.

Liu, Y. "Outside Options and CEO Turnover: The Network Effect." Journal of Corporate Finance, 28 (2014), 201-217.

Morse, A.; V. Nanda; and A. Seru. “Are Incentive Contracts Rigged by Powerful CEOs?” Journal of Finance, 66 (2011), 1779-1821.

Newman, M. E. J. "The Structure and Function of Complex Networks." SIAM Review, 45 (2003), $167-256$.

Nguyen, B. D. "Does the Rolodex Matter? Corporate Elite's Small World and the Effectiveness of Boards of Directors.” Management Science, 58 (2012), 236-252.

Omer, T. C.; M. K. Shelley; and F. M. Tice. "Do Director Networks Matter for Financial Reporting Quality? Evidence from Restatements." Working Paper, University of Nebraska at Lincoln and University of Colorado at Boulder. Available at http://ssrn.com/abstract=2379151 (2016).

PricewaterhouseCoopers. "Achieving Excellence: The Audit Committee's Role in Deterring Fraud," Audit Committee Excellence Series. Available at http://www.corporatecomplianceinsights.com/ wp-content/uploads/2015/12/ACES-Audit-Committees-Role-in-Deterring-Fraud.pdf (2015).

Proctor, C. H., and C. P. Loomis. "Analysis of Sociometric Data." In Research Methods in Social Relations with Special Reference to Prejudice, Vol. 2, M. Jahoda, M. Deutsch, and S. W. Cook, eds. New York, NY: Dryden (1951), 561-585.

Raheja, C. G. "Determinants of Board Size and Composition: A Theory of Corporate Boards." Journal of Financial and Quantitative Analysis, 40 (2005), 283-306.

Sabidussi, G. "The Centrality Index of a Graph." Psychometrika, 31 (1966), 581-603. 
Shivdasani, A., and D. Yermack. "CEO Involvement in the Selection of New Board Members: An Empirical Analysis.” Journal of Finance, 54 (1999), 1829-1853.

Shleifer, A., and R. W. Vishny. "A Survey of Corporate Governance.” Journal of Finance, 52 (1997), 737-783.

Srinivasan, S. "Consequences of Financial Reporting Failure for Outside Directors: Evidence from Accounting Restatements and Audit Committee Members." Journal of Accounting Research, 43 (2005), 291-334.

Teoh, S.; I. Welch; and T. Wong. "Earnings Management and the Subsequent Market Performance of Initial Public Offerings.” Journal of Finance, 53 (1998), 1935-1974.

Wang, C.; F. Xie; and M. Zhu. "Industry Expertise of Independent Directors and Board Monitoring." Journal of Financial and Quantitative Analysis, 50 (2015), 929-962.

Watts, R. "Conservatism in Accounting, Part I: Explanations and Implications." Accounting Horizons, 17 (2003), 207-221.

Xie, H. "The Mispricing of Abnormal Accruals." Accounting Review, 76 (2001), 357-373.

Yermack, D. "Remuneration, Retention, and Reputation Incentives for Outside Directors." Journal of Finance, 59 (2004), 2281-2308. 